\title{
MINIMAL UNIVERSAL METRIC SPACES
}

\author{
Victoriia Bilet, Oleksiy Dovgoshey, \\ Mehmet Küçükaslan and Evgenii Petrov
}

Institute of Applied Mathematics and Mechaniks of NASU, Function Theory Department Dobrovolskogo str. 1, Slovyansk, 84100, Ukraine; victoriiabilet@gmail.com

Institute of Applied Mathematics and Mechaniks of NASU, Function Theory Department Dobrovolskogo str. 1, Slovyansk, 84100, Ukraine; oleksiy.dovgoshey@gmail.com

Mersin University, Faculty of Art and Sciences, Department of Mathematics

Mersin 33342, Turkey; mkucukaslan@mersin.edu.tr

Institute of Applied Mathematics and Mechaniks of NASU, Function Theory Department

Dobrovolskogo str. 1, Slovyansk, 84100, Ukraine; eugeniy.petrov@gmail.com

\begin{abstract}
Let $\mathfrak{M}$ be a class of metric spaces. A metric space $Y$ is minimal $\mathfrak{M}$-universal if every $X \in \mathfrak{M}$ can be isometrically embedded in $Y$ but there are no proper subsets of $Y$ satisfying this property. We find conditions under which, for given metric space $X$, there is a class $\mathfrak{M}$ of metric spaces such that $X$ is minimal $\mathfrak{M}$-universal. We generalize the notion of minimal $\mathfrak{M}$-universal metric space to notion of minimal $\mathfrak{M}$-universal class of metric spaces and prove the uniqueness, up to an isomorphism, for these classes. The necessary and sufficient conditions under which the disjoint union of the metric spaces belonging to a class $\mathfrak{M}$ is minimal $\mathfrak{M}$-universal are found. Examples of minimal universal metric spaces are constructed for the classes of the three-point metric spaces and $n$-dimensional normed spaces. Moreover minimal universal metric spaces are found for some subclasses of the class of metric spaces $X$ which possesses the following property. Among every three distinct points of $X$ there is one point lying between the other two points.
\end{abstract}

\section{Introduction}

Let $X$ and $Y$ be metric spaces. Recall that a function $f: X \rightarrow Y$ is an isometric embedding if the equality $d_{X}(x, y)=d_{Y}(f(x), f(y))$ holds for all $x, y \in X$. In what follows the notation $f: X \hookrightarrow Y$ means that $f$ is an isometric embedding of $X$ in $Y$. We say that $X$ is isometrically embedded in $Y$ and write $X \hookrightarrow Y$ if there exists $f: X \hookrightarrow Y$. The situation when $X \hookrightarrow Y$ does not hold will be denoted as $X \hookrightarrow Y$.

We find it useful to set that the empty metric space is isometrically embedded in every metric space $Y, \varnothing \hookrightarrow Y$. Moreover, $Y \hookrightarrow \varnothing$ holds if and only if $Y=\varnothing$.

Definition 1.1. Let $\mathfrak{M}$ be a class of metric spaces. A metric space $Y$ is said to be universal for $\mathfrak{M}$ or $\mathfrak{M}$-universal if $X \hookrightarrow Y$ holds for every $X \in \mathfrak{M}$.

In what follows the expression $\mathfrak{M} \hookrightarrow Y$ signifies that $Y$ is a $\mathfrak{M}$-universal metric space.

Recall that a class $\mathfrak{A}$ is a set if and only if there is a class $\mathfrak{B}$ such that $\mathfrak{A} \in \mathfrak{B}$. We shall use the capital Gothic letters $\mathfrak{A}, \mathfrak{B}, \ldots$ to denote classes of metric spaces, the capital Roman letters $A, B, \ldots$ to denote metric spaces and the small letters $a, b, \ldots$ for points of these spaces.

https://doi.org/10.5186/aasfm.2017.4261

2010 Mathematics Subject Classification: Primary 54E35, 30L05, 54E40, 51F99.

Key words: Metric space, isometric embedding, universal metric space, betweenness relation in metric spaces. 
It is relevant to remark that throughout this paper we shall make no distinction in notation between a set $X$ and a metric space with a support $X$. For example $X \subseteq Y$ means that $X$ is a subset of a set $Y$ or that $X$ is a subspace of a metric space $Y$ with the metric induced from $Y$.

Definition 1.2. Let $\mathfrak{M}$ be a class of metric spaces and let $Y$ be a $\mathfrak{M}$-universal metric space. The space $Y$ is minimal $\mathfrak{M}$-universal if the implication

$$
\left(\mathfrak{M} \hookrightarrow Y_{0}\right) \Longrightarrow\left(Y_{0}=Y\right)
$$

holds for every subspace $Y_{0}$ of $Y$.

This basic definition was previously used by Holstynski in [20] and [21]. It should be noted here that some other "natural" definitions of minimal universal metric spaces can be introduced. For example instead of (1.1) we can use the implication

$$
\left(\mathfrak{M} \hookrightarrow Y_{0}\right) \Longrightarrow\left(Y \hookrightarrow Y_{0}\right)
$$

or

$$
\left(\mathfrak{M} \hookrightarrow Y_{0}\right) \Longrightarrow\left(Y_{0} \simeq Y\right)
$$

where $Y_{0} \simeq Y$ means that $Y$ and $Y_{0}$ are isometric. It is easy to show that (1.1), (1.2) and (1.3) lead to the three different concepts of minimal universal metric spaces. See, in particular, Proposition 3.17 for an example of a family $\mathfrak{M}$ which has an up to isometry unique, minimal $\mathfrak{M}$-universal metric space if we use (1.2) or (1.3), and which do not admit any minimal $\mathfrak{M}$-universal metric space in the sense of (1.1). In the present paper we mainly consider the minimal universal metric spaces in the sense of Definition 1.2.

The structure of the paper can be described as following.

- The second section is a short survey of some results related to universal metric spaces.

- Sufficient conditions under which a metric space is minimal universal for a class of metric spaces are obtained in Section 3. Moreover, there we find some conditions of non existence of minimal $\mathfrak{M}$-universal metric spaces for given $\mathfrak{M}$.

- The subspaces of minimal universal metric spaces are discussed briefly in Section 4.

- In the fifth section we generalize the notion of minimal M-universal metric space to the notion of minimal $\mathfrak{M}$-universal class of metric spaces. It is proved that a minimal $\mathfrak{M}$-universal class, if it exists, is unique up to an isomorphism.

- In the sixth section we discuss when one can construct a minimal $\mathfrak{M}$-universal metric space using disjoint union of metric spaces belonging to $\mathfrak{M}$.

- Section 7 deals with metric spaces which are minimal universal for some subclasses of the class of metric spaces $X$ that possesses the following property. Among every three distinct points of $X$ there is one point lying between the other two points.

- Two simple examples of universal metric spaces which are minimal for the class of three-point metric spaces are given in Section 8.

\section{A short survey of universal metric spaces}

Let us denote by $\mathfrak{S}$ the class of separable metric spaces. In 1910 Frechet [16] proved that the space $l^{\infty}$ of bounded sequences of real numbers with the sup-norm is $\mathfrak{S}$-universal. This result admits a direct generalization to the class $\mathfrak{S}_{\tau}$ of metric 
spaces of weight at most $\tau$ for an arbitrary cardinal number $\tau$. Indeed, the weight of a metric space $X$ is the smallest cardinal number which is the cardinality of an open base of $X$. The weight of $X$ coincides with the smallest cardinality of dense subsets of $X$. In 1935, Kuratowski [27] proved that every metric space $X$ is isometrically embedded in the space $L^{\infty}(X)$ of bounded real-valued functions on $X$ with sup-norm. The Kuratowski embedding

$$
X \ni x \mapsto f_{x} \in L^{\infty}(X)
$$

was defined as $f_{x}(y)=d_{X}(x, y)-d_{X}\left(x_{0}, y\right)$, where $x_{0}$ is a marked point in $X$. It is evident that for every dense subset $X_{0}$ of $X$ the equality

$$
\sup _{y \in X}\left|f_{x_{1}}(y)-f_{x_{2}}(y)\right|=\sup _{y \in X_{0}}\left|f_{x_{1}}(y)-f_{x_{2}}(y)\right|
$$

holds for all $x_{1}, x_{2} \in X$. Hence if $A$ is a set with $|A|=\tau$, then $L^{\infty}(A)$ is $\mathfrak{S}_{\tau}$-universal. In 1924, Urysohn [41, 42] was the first who gave an example of separable $\mathfrak{S}$ universal metric space (note that $l^{\infty}$ is not separable). In 1986 Katetov [26] proposed an extension of Urysohn's construction to a $\mathfrak{S}_{\tau}$-universal metric space of the weight $\tau=\tau^{<\tau}>\omega$. Recently Uspenskiy [43, 44, 45], followed by Vershik [47, 48, 49] and later Gromov, positioned the Urysohn space with the correspondence to several mathematical disciplines: Functional Analysis, Probability Theory, Dynamics, Combinatorics, Model Theory and General Topology. In 2009 Lešnik gave a "constructive model" of Urysohn space [30]. A computable version of this space was given by Kamo in 2005 [25].

The graphic metric space of the Rado graph [36] (the vertex-set consists of all prime numbers $p \equiv 1(\bmod 4)$ with $p q$ being an edge if $p$ is a quadratic residue modulo $q$ ) is a universal metric space for the class of at most countable metric spaces with distances 0,1 and 2 only.

The Banach-Mazur theorem [2] asserts that the space $C[0,1]$ of continuous functions $f:[0,1] \rightarrow \mathbf{R}$ with the sup-norm is $\mathfrak{S}$-universal. This famous theorem has numerous interesting modifications. As an example we only mention that every separable Banach space is isometrically embedded in the subspace of $C[0,1]$ consisting of nowhere differentiable functions that was proved by Rodriguez-Piazza [37] in 1995.

The following unexpected result was obtained by Holsztynski in 1978 [19]. There exists a with the usual topology compatible metric on $\mathbf{R}$ such that $\mathbf{R}$ with this metric is universal for the class $\mathfrak{F}$ of finite metric spaces. Some interesting examples of minimal universal metric spaces for the classes $\mathfrak{F}_{k}$ of metric spaces $X$ with $|X| \leq k$, $k=2,3,4$, can be found in [20,21].

The class $\mathfrak{S} \mathfrak{U}$ of separable ultrametric spaces is another example of an important class of metric spaces for which the universal spaces are studied in some details. It was proved by Timan and Vestfrid in 1983 [40] that the space $l_{2}$ of real sequences $\left(x_{n}\right)_{n \in \mathbf{N}}$ with the norm $\left(\sum_{n \in \mathbf{N}} x_{n}^{2}\right)^{1 / 2}$ is $\mathfrak{S U}$-universal. The first example of ultrametric space which is universal for $\mathfrak{S} \mathfrak{U}$ was obtained by Vestfrid in 1994 [50]. As was proved by Lemins in [29], if an ultrametric space $Y$ is $\mathfrak{F}_{2}$-universal, then the weight of $Y$ is not less than the continuum $\mathfrak{c}$. Consequently no $\mathfrak{S U}$-universal ultrametric space can be separable. In this connection it should be pointed out that, under some set-theoretic assumptions, for every cardinal $\tau>\mathfrak{c}$ there is an ultrametric space $L W_{\tau} \in \mathfrak{S}_{\tau}$ such that every ultrametric space from $\mathfrak{S}_{\tau}$ can be isometrically embedded into $L W_{\tau}$. The last statement was proved by Vaughan in 1999 [46]. 
There also exist results about spaces which are universal for some classes of compact metric spaces and separable Banach spaces (see, e.g., [15] and [17]). In particular, the necessary and sufficient conditions under which for given family of compact metric spaces there exists a compact universal metric space were found by Iliadis in 1995 [22]. The same author considered also the existence of universal spaces in various subclasses of $\mathfrak{S}[23]$.

\section{Minimal universal metric spaces. Existence, nonexistence and uniqueness}

Let $X$ and $Y$ be metric spaces. Recall that an isometric embedding $f: X \hookrightarrow Y$ is an isometry if $f$ is a surjection. The spaces $X$ and $Y$ are isometric if there is an isometry $f: X \hookrightarrow Y$. We shall write $X \simeq Y$ if $X$ and $Y$ are isometric. Otherwise, we use the notation $X \not Y Y$.

Definition 3.1. A metric space $Y$ is shifted if there is $X \subseteq Y$ such that $X \simeq Y$ and $Y \backslash X \neq \varnothing$. Otherwise, the space $Y$ is said to be unshifted.

It follows directly from the definition, that a non-empty metric space $Y$ is unshifted if and only if every self embedding $f: Y \hookrightarrow Y$ is an isometry.

Example 3.2. All finite metric spaces are unshifted. In particular, the empty metric space is unshifted.

Proposition 3.3. Let $Y$ be a metric space. The following conditions are equivalent.

(i) $Y$ is unshifted.

(ii) $Y$ is minimal universal for the class consisting only of the metric space $Y$.

(iii) There exists a class $\mathfrak{M}$ of metric spaces such that $Y$ is minimal $\mathfrak{M}$-universal.

Proof. It follows directly from the definitions, that (i) $\Longleftrightarrow$ (ii) holds. The implication (ii) $\Longrightarrow$ (iii) is evident. Let us prove (iii) $\Longrightarrow$ (i). Suppose there is a class $\mathfrak{M}$ of metric spaces such that $Y$ is minimal $\mathfrak{M}$-universal. We must show that

$$
f(Y)=Y
$$

holds for every $f: Y \hookrightarrow Y$. Let us consider an arbitrary $f: Y \hookrightarrow Y$. Since $Y$ is $\mathfrak{M}$-universal, the space $f(Y)$ is also $\mathfrak{M}$-universal. Now using (1.1) with $Y_{0}=f(Y)$, we obtain (3.1).

Proposition 3.3 implies that every minimal universal metric space is unshifted. Simple examples show that there is a family $\mathfrak{M}$ of metric spaces and an unshifted metric space $Y$ such that $\mathfrak{M} \hookrightarrow Y$ and $Y$ is not minimal $\mathfrak{M}$-universal. The situation is quite different if, for unshifted $Y$, we assume $\mathfrak{M} \hookrightarrow Y$ and $Y \in \mathfrak{M}$.

Theorem 3.4. Let $\mathfrak{Y}$ be a class of metric spaces, $Y \in \mathfrak{Y}$ and let $\mathfrak{Y} \hookrightarrow Y$. Then the following conditions are equivalent.

(i) $Y$ is unshifted.

(ii) $Y$ is minimal $\mathfrak{Y}$-universal.

(iii) There exists a minimal $\mathfrak{Y}$-universal $X \in \mathfrak{Y}$.

(iv) There exists a minimal $\mathfrak{Y}$-universal metric space.

Proof. Suppose that, on the contrary, (i) holds but $Y$ is not minimal $\mathfrak{Y}$-universal. Then, by Definition 1.2, there is $y_{0} \in Y$ such that $\mathfrak{Y} \hookrightarrow Y \backslash\left\{y_{0}\right\}$. Since $Y \in \mathfrak{Y}$, there exists $f: Y \hookrightarrow Y \backslash\left\{y_{0}\right\}$. Let $i n: Y \backslash\left\{y_{0}\right\} \rightarrow Y$ be the standard injection, in $(y)=y$ 
for every $y \in Y \backslash\left\{y_{0}\right\}$. Then the isometric embedding

$$
Y \stackrel{f}{\longrightarrow} Y \backslash\left\{y_{0}\right\} \stackrel{i n}{\longrightarrow} Y
$$

is not an isometry, contrary to (i). The implication (i) $\Longrightarrow$ (ii) follows. The implications (ii) $\Longrightarrow$ (iii) and (iii) $\Longrightarrow$ (iv) are trivial. Furthermore, Proposition 3.3 implies (ii) $\Longrightarrow$ (i). To complete the proof, it suffices to show that (iv) $\Longrightarrow$ (ii) holds. Let $X$ be a minimal $\mathfrak{Y}$-universal metric space. We claim that $Y$ is also minimal $\mathfrak{Y}$-universal. Indeed, since $Y \in \mathfrak{Y}$ and $\mathfrak{Y} \hookrightarrow X$, there is $X_{0} \subseteq X$ such that $X_{0} \simeq Y$. Now from $\mathfrak{Y} \hookrightarrow Y$ and $X_{0} \simeq Y$ it follows that $\mathfrak{Y} \hookrightarrow X_{0}$. Since $X$ is minimal $\mathfrak{Y}$-universal, the statements $\mathfrak{Y} \hookrightarrow X_{0}$ and $X_{0} \subseteq X$ imply $X_{0}=X$. Hence, $X_{0}$ is minimal $\mathfrak{Y}$-universal. Since $X_{0} \simeq Y$, the metric space $Y$ is also minimal Y)-universal.

Analyzing the proof of Theorem 3.4, we obtain the following.

Proposition 3.5. Let $\mathfrak{M}$ be a class of metric spaces and let $X$ and $Y$ be metric spaces such that $\mathfrak{M} \hookrightarrow X$ and $\mathfrak{M} \hookrightarrow Y$. If $Y$ is minimal $\mathfrak{M}$-universal and $X \in \mathfrak{M}$, then $X \simeq Y$.

Corollary 3.6. (Isometry of minimal universal metric spaces) Let $\mathfrak{Y}$ be a class of metric spaces. If there exists a $\mathfrak{Y}$-universal space $Y \in \mathfrak{Y}$, then every two minimal $\mathfrak{Y}$-universal metric spaces are isometric.

If a class $\mathfrak{Y}$ of metric spaces does not contain any $\mathfrak{Y}$-universal metric space, then we may generally have two minimal $\mathfrak{Y}$-universal metric spaces $W$ and $Z$ such that $W \not Z$.

Example 3.7. Let $\mathfrak{Y}=\{X, Y\}$ with

$$
X=\left\{x_{1}, x_{2}\right\}, \quad d_{X}\left(x_{1}, x_{2}\right)=1 \quad \text { and } \quad Y=\left\{y_{1}, y_{2}\right\}, \quad d_{Y}\left(y_{1}, y_{2}\right)=2
$$

and let $1<a<b<2$. The metric spaces

$$
Z=\left\{z_{1}, z_{2}, z_{3}\right\}, \quad W=\left\{w_{1}, w_{2}, w_{3}\right\}
$$

with

$$
\begin{aligned}
& d_{Z}\left(z_{1}, z_{2}\right)=d_{W}\left(w_{1}, w_{2}\right)=1, \quad d_{Z}\left(z_{2}, z_{3}\right)=d_{W}\left(w_{2}, w_{3}\right)=2, \\
& d_{Z}\left(z_{1}, z_{3}\right)=a, \quad d_{W}\left(w_{1}, w_{3}\right)=b
\end{aligned}
$$

are minimal $\mathfrak{Y}$-universal. It is clear that $Z \not W$.

In the fifth section of the paper we shall show that the nonuniqueness of minimal $\mathfrak{M}$-universal metric spaces can be overcome by extending the concept of a minimal $\mathfrak{M}$ universal metric space to the concept of minimal $\mathfrak{M}$-universal class of metric spaces (see Definition 5.3 and Theorem 5.18).

Proposition 3.8. (Nonexistence of minimal universal spaces) Let $\mathfrak{M}$ be a class of non-empty metric spaces. The class $\mathfrak{M}$ admits no minimal universal metric spaces if at least one from the following conditions holds.

(i) There are $X, Y \in \mathfrak{M}$ such that

$$
\mathfrak{M} \hookrightarrow X, \quad \mathfrak{M} \hookrightarrow Y \quad \text { and } X \not 4 Y \text {. }
$$

(ii) For every $X \in \mathfrak{M}$ there exist metric spaces $Y, Y_{1}$ and $Y_{2}$ which satisfy the conditions

$$
Y \in \mathfrak{M}, \quad Y_{1} \subseteq Y, \quad Y_{2} \subseteq Y, \quad Y_{1} \cap Y_{2}=\varnothing
$$


and

$$
Y_{1} \simeq X \simeq Y_{2}
$$

Proof. It is an immediate consequence of Proposition 3.5 that (i) implies the nonexistence of minimal $\mathfrak{M}$-universal metric spaces. Let us consider (ii).

Suppose that $W$ is a minimal $\mathfrak{M}$-universal metric space and (ii) holds. Let $w_{0} \in W$ and

$$
W_{0}=W \backslash\left\{w_{0}\right\} .
$$

Since $W$ is minimal $\mathfrak{M}$-universal, there is $X \in \mathfrak{M}$ such that $X \hookrightarrow W_{0}$. Using (ii) we can find metric spaces $Y, Y_{1}$ and $Y_{2}$ which satisfy (3.2) and (3.3). From $Y \hookrightarrow W$ and (3.2) it follows that there exist $W_{1} \subseteq W$ and $W_{2} \subseteq W$ such that

$$
W_{1} \simeq Y_{1}, \quad W_{2} \simeq Y_{2} \quad \text { and } \quad W_{1} \cap W_{2}=\varnothing .
$$

The last equality implies $W_{1} \cap\left\{w_{0}\right\}=\varnothing$ or $W_{2} \cap\left\{w_{0}\right\}=\varnothing$. We may assume, without loss of generality, that $W_{1} \cap\left\{w_{0}\right\}=\varnothing$. Hence, $W_{1} \subseteq W_{0}$ holds. It follows from (3.3) and (3.4) that $X \simeq W_{1}$. Consequently, we have $X \hookrightarrow W_{0}$, which is a contradiction.

Remark 3.9. Proposition 3.8 remains valid if we suppose that there is a nonempty $Y \in \mathfrak{M}$ instead of $X \neq \varnothing$ for all $X \in \mathfrak{M}$. If $\mathfrak{M}=\{\varnothing\}$, then $\mathfrak{M}$ satisfies condition (ii) of Proposition 3.8 and $X=\varnothing$ is minimal $\mathfrak{M}$-universal.

Example 3.10. Let $\mathfrak{F}$ be the class of finite non-empty metric spaces. Condition (ii) of Proposition 3.8 is valid for $\mathfrak{M}=\mathfrak{F}$. Consequently, by Proposition 3.8, the class $\mathfrak{F}$ does not admit any minimal $\mathfrak{F}$-universal metric spaces. In particular, the Holsztynski metric space (see [19]) is $\mathfrak{F}$-universal, but not minimal $\mathfrak{F}$-universal.

In Example 3.10 instead of $\mathfrak{F}$ we can take the class of finite metric subspaces of the usual real line $\mathbf{R}$. Some other examples of families $\mathfrak{M}$, which do not admit minimal $\mathfrak{M}$-universal metric spaces will be given after the corresponding lemmas.

Recall that a metric space $X$ is ultrametric if the strong triangle inequality

$$
d_{X}(x, y) \leq \max \left\{d_{X}(x, z), d_{X}(z, y)\right\}
$$

holds for all $x, y, z \in X$.

Lemma 3.11. Let $X$ and $Y$ be disjoint ultrametric spaces, let $x_{0} \in X$ and $y_{0} \in Y$, and let $r_{0}$ be a positive real number. Then there is an ultrametric $d_{Z}$ on $Z=X \cup Y$ such that

$$
d_{Z}(x, y)= \begin{cases}d_{X}(x, y) & \text { if } x, y \in X \\ d_{Y}(x, y) & \text { if } x, y \in Y, \\ r_{0} & \text { if } x=x_{0} \text { and } y=y_{0} .\end{cases}
$$

Proof. It is a particular case of Theorem 2 from [10].

Lemma 3.12. Let $r_{0} \in(0, \infty)$ and let $X_{1}$ and $X_{2}$ be disjoint non-empty metric spaces with

$$
r_{0} \geq \max \left\{\operatorname{diam} X_{1}, \operatorname{diam} X_{2}\right\}>0,
$$

where

$$
\operatorname{diam} X_{i}=\sup \left\{d_{X_{i}}(x, y): x, y \in X_{i}\right\}, \quad i=1,2 .
$$


Then the function

$$
d_{Z}(x, y)= \begin{cases}d_{X_{1}}(x, y) & \text { if } x, y \in X_{1}, \\ d_{X_{2}}(x, y) & \text { if } x, y \in X_{2}, \\ r_{0} & \text { if } x \in X_{1}, y \in X_{2} \text { or } x \in X_{2}, y \in X_{1},\end{cases}
$$

is a metric on $Z=X_{1} \cup X_{2}$.

A proof is simple, so that we omit it here.

Example 3.13. Let $\tau$ be an infinite cardinal number and $\mathfrak{S}_{\tau} \mathfrak{U}$ be the class of ultrametric spaces of weight at most $\tau$. If

$$
X, Y \in \mathfrak{S}_{\tau} \mathfrak{U}, X \cap Y=\varnothing, x_{0} \in X, y_{0} \in Y, r_{0} \in(0, \infty), Z=X \cup Y
$$

and $d_{Z}$ satisfies (3.5), then the weight of $Z$ is at most $\tau$. Hence, condition (ii) of Proposition 3.8 holds with $\mathfrak{M}=\mathfrak{S}_{\tau} \mathfrak{U}$. Consequently, $\mathfrak{S}_{\tau} \mathfrak{U}$ admits no minimal $\mathfrak{S}_{\tau} \mathfrak{U}$-universal metric spaces. In particular, the ultrametric space $L W_{\tau}$, which was considered by A. Lemin and V. Lemin in [29], is $\mathfrak{S}_{\tau} \mathfrak{U}$-universal, but not minimal $\mathfrak{S}_{\tau} \mathfrak{U}$-universal.

The next example shows that the class $\mathfrak{S}$ of separable metric spaces does not admit any minimal universal metric spaces.

Example 3.14. Let $\bar{C}[0,1]$ be the metric subspace of $C[0,1]$ consisting of nowhere differentiable functions. Since $C[0,1]$ is complete and $\bar{C}[0,1]$ is incomplete, we have $C[0,1] \not \bar{C}[0,1]$. Using the Banach-Mazur theorem, the theorem of RodrieguesPiazza and Proposition 3.8 with $\mathfrak{M}=\mathfrak{S}$, we see that there are no minimal $\mathfrak{S}$-universal metric spaces.

Example 3.15. Let $\mathfrak{D}_{3}$ be the class of all at most countable metric spaces with the distance sets in $\{0,1,2\}$. Using condition (ii) of Propositon 3.8 and defining $d_{Z}$ by (3.6) with $r_{0}=2$ for disjoint $X, Y \in \mathfrak{D}_{3}$, we see that $\mathfrak{D}_{3}$ admits no minimal universal metric spaces. Consequently, the graphic metric space of the Rado graph [36] is $\mathfrak{D}_{3}$-universal, but not minimal $\mathfrak{D}_{3}$-universal.

Recall that a metric $d_{X}$ on $X$ is discrete if the equality $d_{X}(x, y)=1$ holds for all distinct $x, y \in X$.

Example 3.16. Let $\tau$ be an infinite cardinal number. Write $\mathfrak{M D}^{\tau}$ for the class of metric spaces $X$ with $|X| \leq \tau$ and discrete $d_{X}$. It is clear that:

- $(X \simeq Y) \Longleftrightarrow(|X|=|Y|)$ holds for all $X, Y \in \mathfrak{M D}^{\tau}$;

- A metric space $X \in \mathfrak{M D}^{\tau}$ is $\mathfrak{M D}^{\tau}$-universal if and only if $|X|=\tau$;

- There are no minimal $\mathfrak{M D}^{\tau}$-universal metric spaces.

Proposition 3.17. If $Y \in \mathfrak{M D}^{\tau}$, then the implication

$$
\left(\mathfrak{M D}^{\tau} \hookrightarrow Y_{0}\right) \Longrightarrow\left(Y_{0} \simeq Y\right)
$$

holds for every subspace $Y_{0}$ of $Y$. Moreover, if $Y$ is an arbitrary metric space and (3.7) holds for every $Y_{0} \subseteq Y$, then $Y \in \mathfrak{M D}^{\tau}$ and $|Y|=\tau$.

A simple proof is omitted here.

Remark 3.18. As in Example 3.13 we can show that the class $\mathfrak{S}_{\tau}$ with $\tau>\omega$ does not admit any minimal universal metric spaces. Thus the Katetov space is not minimal $\mathfrak{S}_{\tau}$-universal. 
We now turn to some conditions under which the metric spaces are unshifted. The following lemma seems to be known up to the terminology.

Lemma 3.19. Every compact metric space is unshifted.

Proof. Let $X$ be a compact metric space and let $f: X \hookrightarrow X$ be an isometric self embedding. Suppose that a point $p$ belongs to $X \backslash f(X)$. Define a sequence $\left(p_{i}\right)_{i \in \mathbf{N}}$ in $X$ as

$$
p_{1}=p, p_{2}=f\left(p_{1}\right), p_{3}=f\left(p_{2}\right), \text { ldots }
$$

and so on. Since $f$ is continuous, $f(X)$ is a compact subset of $X$. Hence the number

$$
\varepsilon_{0}:=\inf _{y \in f(X)} d_{X}\left(p_{1}, y\right)
$$

is strictly positive, $\varepsilon_{0}>0$. It follows from (3.8), that $p_{i} \in f(X)$ for every $i \geq 2$. Consequently, the inequality $d_{X}\left(p_{1}, p_{i}\right) \geq \varepsilon_{0}$ holds for every $i \geq 2$. The function $f$ preserves the distances. Hence, for $i>j$, we have

$$
d_{X}\left(p_{j}, p_{i}\right)=d_{X}\left(f\left(p_{j-1}\right), f\left(p_{i-1}\right)\right)=d_{X}\left(p_{j-1}, p_{i-1}\right)=\ldots=d_{X}\left(p_{1}, p_{i-(j-1)}\right) \geq \varepsilon_{0} .
$$

Thus, $d_{X}\left(p_{i}, p_{j}\right) \geq \varepsilon_{0}>0$ if $i \neq j$. In particular, the sequence $\left(p_{i}\right)_{i \in \mathbf{N}}$ has not any convergent subsequence, contrary to the compactness of $X$. This contradiction implies $f(X)=X$. By Definition 3.1, $X$ is unshifted.

Lemma 3.19 and Theorem 3.4 give us the following result (cf. Proposition 3.17).

Theorem 3.20. Let $\mathfrak{Y}$ be a class of compact metric spaces. Then the following conditions are equivalent.

(i) There is a minimal $\mathfrak{Y}$-universal metric space belonging to $\mathfrak{Y}$.

(ii) There is a $\mathfrak{Y}$-universal metric space belonging to $\mathfrak{Y}$.

In Proposition 7.16 of the paper we construct a family $\mathfrak{M}$ of metric spaces such that:

- All $X \in \mathfrak{M}$ are compact;

- There is a compact $\mathfrak{M}$-universal metric space;

- There is at least one minimal $\mathfrak{M}$-universal metric space;

- If $X$ and $Y$ are minimal $\mathfrak{M}$-universal, then $X$ and $Y$ are not compact and $X \simeq Y$.

Recall that a metric space $X$ is boundedly compact if all closed bounded subspaces of $X$ are compact. We can prove an analogue of Lemma 3.19 for some boundedly compact metric spaces.

Definition 3.21. A metric space $X$ is homogeneous if, for every pair $x, y \in X$, there is $f: X \hookrightarrow X$ such that $f(x)=y$.

Remark 3.22. Definition 3.21 is slightly non-standard. Usually we say that a metric space $X$ is homogenous if its isometry group $\operatorname{Iso}(X)$ acts transitively on points. The following lemma shows, in particular, that the standard definition and Definition 3.21 are equivalent for boundedly compact $X$.

Lemma 3.23. All boundedly compact homogeneous metric spaces are unshifted.

Proof. Let $X$ be a boundedly compact homogeneous metric space. We must show that $f(X)=X$ for every $f: X \hookrightarrow X$. Let $p_{0} \in X$. Write $p_{1}=f\left(p_{0}\right)$. Since $X$ is homogeneous there is $g: X \hookrightarrow X$ such that $g\left(p_{1}\right)=p_{0}$. Since $g$ is an injective 
function, the equality $f(X)=X$ holds if

$$
g(f(X))=X .
$$

It is easy to prove that the last equality holds if

$$
g\left(f\left(B_{r}\left(p_{0}\right)\right)\right)=B_{r}\left(p_{0}\right)
$$

for every ball $B_{r}\left(p_{0}\right)=\left\{x: d_{X}\left(p_{0}, x\right) \leq r\right\}$. The function $g \circ f$ is an isometric self embedding of $X$. Since $p_{0}$ is a fixed point of $g \circ f$, the restriction $\left.g \circ f\right|_{B_{r}\left(p_{0}\right)}$ is an isometric self embedding of the compact metric space $B_{r}\left(p_{0}\right)$. Now (3.9) follows from Lemma 3.19.

Just as in Lemma 3.23 we can prove the following proposition.

Proposition 3.24. Let $X$ be a boundedly compact metric space. If for every $f: X \hookrightarrow X$ there is a fixed point $x_{0} \in X, f\left(x_{0}\right)=x_{0}$, then $X$ is unshifted.

Remark 3.25. If $X$ is a finite-dimensional normed linear space over the field $\mathbf{R}$ or the field $\mathbf{C}$, then $X$ is unshifted because such spaces are homogeneous and boundedly compact. This fact is well known. In particular in the high school geometry, the isometries of the plane $\mathbf{R}^{2}$ are usually defined as mappings $f: \mathbf{R}^{2} \rightarrow \mathbf{R}^{2}$ which preserve distances. As was noted by Lang [28, p. 31]: "It is not immediately clear that an isometry has an inverse". Next in [28] it is indicated that the existence of the inverses follows from the representation of any isometry by a composition of reflections through some straight lines in $\mathbf{R}^{2}$. Thus Lemma 3.23 can be considered as a generalization of this elementary fact.

The hyperbolic plane $\mathbf{H}$ gives an important example of boundedly compact homogenous metric space. The key point here that in the upper half-plane model of $\mathbf{H}$, the isometries of $\mathbf{H}$ can be identified with elements of the subgroup $\operatorname{Möb}(\mathbf{H})$ of the general Möbius group and $\operatorname{Möb}(\mathbf{H})$ acts transitively on $\mathbf{H}$ (see [1] for details).

Using Lemma 3.23, Theorem 3.4, Corollary 3.6 and Proposition 3.5 we obtain the following.

Theorem 3.26. Let $\mathfrak{M}$ be a class of metric spaces. If $X \in \mathfrak{M}$ is boundedly compact, homogeneous and $\mathfrak{M}$-universal, then $X$ is minimal $\mathfrak{M}$-universal and $Y \simeq X$ holds for all minimal $\mathfrak{M}$-universal $Y$ and all $\mathfrak{M}$-universal $Y \in \mathfrak{M}$.

Proposition 3.27. Let $\mathfrak{M}$ be a class of metric spaces and let $X$ be a homogeneous metric space. If $X$ is minimal $\mathfrak{M}$-universal, then there is $Y \in \mathfrak{M}$ such that $X \simeq Y$.

Proof. Suppose $X$ is $\mathfrak{M}$-universal and $X \not 千 Y$ holds for every $Y \in \mathfrak{M}$. We must show that $X$ is not minimal $\mathfrak{M}$-universal. By the supposition, for every $Y \in \mathfrak{M}$, there are $f: Y \hookrightarrow X$ and $x_{f} \in X$ such that

$$
f(Y) \subseteq X \backslash\left\{x_{f}\right\} .
$$

Let $a$ be a point of $X$. Since $X$ is homogeneous, there is $g: X \hookrightarrow X$ such that $g\left(x_{f}\right)=a$. The function

$$
Y \stackrel{f}{\longrightarrow} X \stackrel{g}{\longrightarrow} X
$$

is an isometric embedding of $Y$ in $X$ for which

$$
g(f(X)) \subseteq X \backslash\{a\} .
$$

Consequently, we have $\mathfrak{M} \hookrightarrow X \backslash\{a\}$. Thus $X$ is not minimal $\mathfrak{M}$-universal, that is a contradiction. 
Corollary 3.28. Let $\mathfrak{M}$ be a class of metric spaces and let $X$ be a homogeneous metric space. If $X$ is minimal $\mathfrak{M}$-universal, then $X \simeq Y$ holds for every minimal $\mathfrak{M}$-universal metric space $Y$.

The so-called strongly rigid metric spaces give us another subclass of unshifted metric spaces.

Definition 3.29. ([24]) A metric space $X$ is said to be strongly rigid if, for all $x, y, u, v \in X$,

imply that $\{x, y\}=\{u, v\}$.

$$
d_{X}(x, y)=d_{X}(u, v) \quad \text { and } \quad x \neq y
$$

Lemma 3.30. Every strongly rigid metric space is unshifted.

Proof. Let $X$ be a strongly rigid metric space. This is clear that $X$ is unshifted if $|X| \leq 1$. Suppose that $|X| \geq 2$. For every $f: X \hookrightarrow X$ the equality

$$
d_{X}(x, y)=d_{X}(f(x), f(y))
$$

holds for all $x, y \in X$. From Definition 3.29 it follows that

$$
\{x\} \subseteq\{x, y\}=\{f(x), f(y)\} \subseteq f(X)
$$

hold for all distinct $x, y \in X$. It follows that $x \in f(X)$ holds for every $x \in X$ and every $f: X \hookrightarrow X$. Hence, every self embedding $f: X \hookrightarrow X$ is a surjection and, therefore, is an isometry.

Definition 3.31. A metrizable space $X$ is said to be eventually strongly rigid if there is a with the topology of $X$ compatible metric $d_{X}$ such that $\left(X, d_{X}\right)$ is strongly rigid. (See $[24,31]$.)

Lemma 3.32. [31] Let $X$ be a non-empty metrizable space with covering dimension zero, $\operatorname{dim}(X)=0$. If $|X| \leq \mathfrak{c}$, where $\mathfrak{c}$ is the cardinality of continuum, then $X$ is eventually strongly rigid.

In analogy with Definition 3.31, we shall say that a metrizable space $X$ is eventually unshifted if there is a with the topology of $X$ compatible metric $d_{X}$ such that $\left(X, d_{X}\right)$ is unshifted.

Lemma 3.30 and Lemma 3.32 imply the following proposition.

Proposition 3.33. Let $X$ be a non-empty metrizable space with $\operatorname{dim}(X)=0$. If $|X| \leq \mathfrak{c}$, then $X$ is eventually unshifted.

\section{Unshifted metric subspaces of minimal universal metric spaces}

For a metric space $X$ denote by $\operatorname{Iso}(X)$ the group of all isometries of $X$. In what follows we do not presuppose that $\operatorname{Iso}(X)$ is equipped with any topology.

Theorem 4.1. Let $X$ be an unshifted and complete metric space. Then the following statements hold.

(i) If for every $g \in \operatorname{Iso}(X)$ and every $x \in X$ there is a positive integer number $m$ such that the equality

$$
g^{m}(x)=x
$$

holds, then every dense subset of $X$ is unshifted.

(ii) If $X$ contains no isolated points and all dense subsets of $X$ are unshifted, then for every $g \in \operatorname{Iso}(X)$ and every $x \in X$, there is a positive integer number $m$ such that (4.1) holds. 
Proof. (i) Suppose that the condition of statement (i) holds. Let $Y$ be a dense subset of $X$ and let $f$ be an isometric self embedding of $Y$. We must show that $f \in \operatorname{Iso}(Y)$. Since $X$ is unshifted, $f(Y)$ is a dense subset of $X$ (otherwise, we would obtain a non-surjective isometric embedding of $X$ into itself). Thus, $f$ extends to an isometry $g: X \rightarrow X$. It follows that $g \in \operatorname{Iso}(X)$ because $X$ is unshifted. Suppose now that $f \notin \operatorname{Iso}(Y)$. Then the set $Y \backslash f(Y)$ is not empty. Let us consider an arbitrary point $x \in Y \backslash f(Y)$. Let $m$ be a positive integer number such that

$$
g^{m}(x)=x .
$$

It is clear that

$$
f^{m}(x) \in f^{m}(Y) \subseteq f^{m-1}(Y) \subseteq \ldots \subseteq f(Y) .
$$

Since $\left.g\right|_{Y}=f$, (4.2) implies $x=f^{m}(x)$. Hence $x \in f(Y)$, contrary to $x \in Y \backslash f(Y)$.

(ii) Suppose now that $X$ contains no isolated points and all dense subsets of $X$ are unshifted. If $g \in \operatorname{Iso}(X), x_{0} \in X$ and $g^{m}\left(x_{0}\right) \neq x_{0}$ for every $m \in \mathbf{N}$, then $g^{n_{1}}\left(x_{0}\right) \neq g^{n_{2}}\left(x_{0}\right)$ for all distinct $n_{1}, n_{2} \in \mathbf{Z}$. Write

$$
A:=\left\{x \in X: x=g^{-n}\left(x_{0}\right), n \in \mathbf{N}\right\} .
$$

The set $X \backslash A$ is a dense subset of $X$. Indeed, we have

$$
X \backslash A=\bigcap_{n=1}^{\infty}\left(X \backslash\left\{g^{-n}\left(x_{0}\right)\right\}\right)
$$

where $\left\{g^{-n}\left(x_{0}\right)\right\}$ is the one point set consisting of the unique point $g^{-n}\left(x_{0}\right)$. Since $X$ has no isolated points, the sets $X \backslash\left\{g^{-n}\left(x_{0}\right)\right\}$ are open dense subsets of $X$. Using the Baire category theorem we obtain that $X \backslash A$ is also dense in $X$. Let $p$ be an arbitrary point of $X \backslash A$. It is easy to show that $g(p) \in X \backslash A$. Indeed, if $g(p) \notin X \backslash A$, then there is $n \in \mathbf{N}$ such that

$$
g(p)=g^{-n}\left(x_{0}\right)
$$

Consequently, we obtain $p=g^{-n-1}\left(x_{0}\right)$. Hence $p$ belongs to $A$, contrary to $p \in X \backslash A$. Thus the restriction $\left.g\right|_{X \backslash A}$ is an isometric self embedding of $X \backslash A$. Let us prove the statement

$$
x_{0} \notin g(X \backslash A) \text {. }
$$

Suppose $t \in(X \backslash A)$ and $g(t)=x_{0}$ hold. The last equality implies $t=g^{-1} x_{0}$. Hence, by the definition of $X \backslash A$, we have $t \in A$. This contradicts $t \in X \backslash A$, so that (4.3) holds. Since (4.3) implies

$$
\left.g\right|_{X \backslash A} \notin \operatorname{Iso}(X \backslash A),
$$

the metric space $X \backslash A$ is unshifted. Statement (ii) follows.

Recall that a group $G$ is torsion if for every $g \in G$ there is $n \in \mathbf{N}$ such that $g^{n}=e$ where $e$ is the identity element of $G$.

Corollary 4.2. Let $X$ be unshifted and complete. If the isometry group $\operatorname{Iso}(X)$ is torsion, then every dense subset of $X$ is unshifted.

Let $X$ be an unshifted and complete metric space without isolated points. Suppose that there are $x \in X$ and $g \in \operatorname{Iso}(X)$ such that $g^{m}(x)=x$ holds if and only if $m=0$. Then Theorem 4.1 implies the existence of $A \subseteq X$ which is shifted and dense (in $X$ ). Let us consider an example of such spaces. 
Example 4.3. Let $\mathbf{S}=\{z \in \mathbf{C}:|z|=1\}$ be the unit circle in the complex plane C. It is clear that $\mathbf{S}$ is a compact subset of $\mathbf{C}$ without isolated points. By Lemma 3.19 the metric space $\mathbf{S}$ is unshifted. An irrational rotation is a map $T_{\theta}: \mathbf{S} \rightarrow \mathbf{S}$ with

$$
T_{\theta}(z)=z e^{2 \pi i \theta}
$$

where $\theta$ is an irrational number. Every irrational rotation $T_{\theta}$ is an isometry of $\mathbf{S}$ and, in addition,

$$
T_{\theta}^{m}(z)=z e^{2 \pi m i}=z
$$

holds if and only if $m=0$. By Theorem 4.1 there is $A \subseteq \mathbf{S}$ which is shifted and dense in $\mathbf{S}$. In fact the set

$$
A=\left\{T_{\theta}^{n}(z): n \in \mathbf{N}\right\}
$$

is dense and shifted for every $z \in \mathbf{S}$.

The previous example is fundamental in the theory of dynamical systems. Using this example we can simply show that the balls and spheres in the finite-dimensional Euclidean spaces have some dense shifted subsets. In the converse direction we have the following proposition.

Proposition 4.4. There is an unshifted metric space $X$ with $|X|=\mathfrak{c}$ such that every subspace $Y$ of $X$ is also unshifted.

Proof. It follows from Lemma 3.30 and Lemma 3.32.

\section{Minimal universality of classes of metric spaces}

Let $\mathfrak{M}$ and $\mathfrak{B}$ be classes of metric spaces. We write $\mathfrak{M} \hookrightarrow \mathfrak{B}$ if for every $X \in \mathfrak{M}$ there is $Y \in \mathfrak{B}$ such that $X \hookrightarrow Y$. The notation $\mathfrak{M} \preceq \mathfrak{B}$ means that, for every $X \in \mathfrak{M}$, there is $Y \in \mathfrak{B}$ such that $X \subseteq Y$. It is easy to see that $\mathfrak{M} \subseteq \mathfrak{B}$ implies $\mathfrak{M} \preceq \mathfrak{B}$ but not conversely.

Definition 5.1. Let $X$ and $Y$ be metric spaces. If $X \hookrightarrow Y$ or $Y \hookrightarrow X$, then $X$ and $Y$ are said to be comparable. Otherwise, $X$ and $Y$ are incomparable.

The following two definitions are similar to Definition 1.1 and Definition 1.2 respectively.

Definition 5.2. Let $\mathfrak{M}$ be a class of metric spaces. A class $\mathfrak{A}$ of metric spaces is said to be universal for $\mathfrak{M}$ or $\mathfrak{M}$-universal if $\mathfrak{M} \hookrightarrow \mathfrak{A}$.

Definition 5.3. Let $\mathfrak{M}$ and $\mathfrak{A}$ be classes of metric spaces. The class $\mathfrak{A}$ is minimal $\mathfrak{M}$-universal if the following conditions hold:

(i) $\mathfrak{A}$ is $\mathfrak{M}$-universal;

(ii) The implication

$$
(\mathfrak{M} \hookrightarrow \mathfrak{B}) \wedge(\mathfrak{B} \preceq \mathfrak{A}) \Rightarrow(\mathfrak{A} \preceq \mathfrak{B})
$$

holds for every class $\mathfrak{B}$ of metric spaces;

(iii) Distinct elements of $\mathfrak{A}$ are incomparable metric spaces.

Proposition 5.4. Let $\mathfrak{N}, \mathcal{P}$ and $\mathfrak{A}$ be classes of metric spaces. If $\mathfrak{A}$ is minimal $\mathfrak{N}$-universal, and

$$
\mathfrak{N} \hookrightarrow \mathcal{P} \quad \text { and } \quad \mathcal{P} \hookrightarrow \mathfrak{N}
$$

then $\mathfrak{A}$ is also minimal $\mathcal{P}$-universal.

Proof. Suppose that $\mathfrak{A}$ is minimal $\mathfrak{N}$-universal and $\mathfrak{N} \hookrightarrow \mathcal{P}$ and $\mathcal{P} \hookrightarrow \mathfrak{N}$. We must show that conditions (i)-(iii) of Definition 5.3 hold with $\mathfrak{M}=\mathcal{P}$. 
(i) Since $\mathfrak{A}$ is minimal $\mathfrak{N}$-universal, we have $\mathfrak{N} \hookrightarrow \mathfrak{A}$, that, together with $\mathcal{P} \hookrightarrow \mathfrak{N}$, implies $\mathcal{P} \hookrightarrow \mathfrak{A}$.

(ii) Let $\mathfrak{B}$ be an arbitrary class of metric spaces. Since condition (ii) holds with $\mathfrak{M}=\mathfrak{N}$, we have

$$
(\mathfrak{N} \hookrightarrow \mathfrak{B}) \wedge(\mathfrak{B} \preceq \mathfrak{A}) \Longrightarrow(\mathfrak{A} \preceq \mathfrak{B}) .
$$

From $\mathfrak{N} \hookrightarrow \mathcal{P}$ it follows that

$$
(\mathcal{P} \hookrightarrow \mathfrak{B}) \wedge(\mathfrak{B} \preceq \mathfrak{A}) \Longrightarrow(\mathfrak{N} \preceq \mathfrak{B}) \wedge(\mathfrak{B} \preceq \mathfrak{A}) .
$$

The last implication together with (5.2) give us (5.1) with $\mathfrak{M}=\mathcal{P}$.

(iii) Condition (iii) does not depend from the choice of class $\mathfrak{M}$ in Definition 5.3. Hence (iii) automatically holds.

Remark 5.5. Instead of condition (ii) we can use the following statement:

(ii*) The implication

$$
(\mathfrak{M} \hookrightarrow \mathfrak{B}) \wedge(\mathfrak{B} \preceq \mathfrak{A}) \Longrightarrow(\mathfrak{A} \subseteq \mathfrak{B})
$$

holds for every class $\mathfrak{B}$ of metric spaces.

For the proof of Remark 5.5 we need the following lemma.

Lemma 5.6. Let $\mathfrak{M}$ and $\mathfrak{N}$ be classes such that $\mathfrak{M} \preceq \mathfrak{N}$ and $\mathfrak{N} \preceq \mathfrak{M}$. Suppose the implication

$$
(A \subseteq C) \Longrightarrow(A=C)
$$

holds for all $A, C \in \mathfrak{M}$. Then we have the inclusion $\mathfrak{M} \subseteq \mathfrak{N}$.

Proof. Let $A \in \mathfrak{M}$. Since $\mathfrak{M} \preceq \mathfrak{N}$, there is $B \in \mathfrak{N}$ such that $A \subseteq B$. From $\mathfrak{N} \preceq \mathfrak{M}$ it follows that there is $C \in \mathfrak{M}$ satisfying $B \subseteq C$. Thus, we have

$$
A \subseteq B \subseteq C
$$

and $A, C \in \mathfrak{M}$. Using (5.4) we see that $A=C$. This equality and (5.5) imply $A=B$. Consequently, for every $A \in \mathfrak{M}$ there is $B \in \mathfrak{N}$ such that $A=B$, i.e., $\mathfrak{M} \subseteq \mathfrak{N}$.

Proof of Remark 5.5. Note that (ii*) $\Rightarrow$ (ii) is valid, because we have

$$
(\mathfrak{A} \subseteq \mathfrak{B}) \Longrightarrow(\mathfrak{A} \preceq \mathfrak{B})
$$

for arbitrary $\mathfrak{A}$ and $\mathfrak{B}$. Moreover, condition (iii) of Definition 5.3 gives us (5.4) for $A, C \in \mathfrak{A}$. Using Lemma 5.6, we obtain that $(($ iii $) \wedge($ ii $)) \Longrightarrow\left(\right.$ ii $\left.^{*}\right)$.

Corollary 5.7. Let $\mathfrak{M}$ and $\mathfrak{A}$ be classes of metric spaces and let $\mathfrak{A}$ be minimal $\mathfrak{M}$-universal. Then the implication

$$
(\mathfrak{M} \hookrightarrow \mathfrak{B}) \wedge(\mathfrak{B} \subseteq \mathfrak{A}) \Longrightarrow(\mathfrak{B}=\mathfrak{A})
$$

holds for every class $\mathfrak{B}$ of metric spaces.

Proof. Let $\mathfrak{B}$ be a class of metric spaces. Then, as was shown above, implication (5.3) holds. Since we evidently have

$$
(\mathfrak{M} \hookrightarrow \mathfrak{B}) \wedge(\mathfrak{B} \subseteq \mathfrak{A}) \Longrightarrow(\mathfrak{M} \hookrightarrow \mathfrak{B}) \wedge(\mathfrak{B} \preceq \mathfrak{A})
$$

using (5.3), we obtain

$$
(\mathfrak{M} \hookrightarrow \mathfrak{B}) \wedge(\mathfrak{B} \subseteq \mathfrak{A}) \Longrightarrow(\mathfrak{A} \subseteq \mathfrak{B}) .
$$

The last implication and the trivial implication

$$
(\mathfrak{M} \hookrightarrow \mathfrak{B}) \wedge(\mathfrak{B} \subseteq \mathfrak{A}) \Longrightarrow(\mathfrak{B} \subseteq \mathfrak{A})
$$


give (5.6).

Let us describe the "degenerate" minimal universal classes of metric spaces.

Proposition 5.8. Let $\mathfrak{M}$ be a non-empty class of metric spaces and let $\mathfrak{A}$ be a minimal $\mathfrak{M}$-universal class of metric spaces. The following statements hold.

(i) We have $\varnothing \in \mathfrak{A}$ if and only if $\mathfrak{A}=\mathfrak{M}=\{\varnothing\}$.

(ii) The following conditions are equivalent:

(ii 1 ) There is $A \in \mathfrak{A}$ such that $|A|=1$;

$\left(\mathrm{ii}_{2}\right)$ The equality $\mathfrak{A}=\{A\}$ holds with some $A$ having $|A|=1$;

(ii $\left.{ }_{3}\right)$ The inequality $|X| \leq 1$ holds for every $X \in \mathfrak{M}$ and, in addition, there is $Y \in \mathfrak{M}$ such that $|Y|=1$.

Proof. Let $\varnothing \in \mathfrak{A}$ and $A \in \mathfrak{A}$. Since $\varnothing \hookrightarrow A$, condition (iii) of Definition 5.3 implies the equality $\varnothing=A$. The equality $\mathfrak{A}=\{\varnothing\}$ follows. If $X$ is an arbitrary metric space belonging to $\mathfrak{M}$, then from $\mathfrak{M} \hookrightarrow \mathfrak{A}$ and $\mathfrak{A}=\{\varnothing\}$ it follows that $X \hookrightarrow \varnothing$, thus $X=\varnothing$. The last equality holds for every $X \in \mathfrak{M}$, i.e. $\mathfrak{M}=\{\varnothing\}$. The statement (i) is proved. Statement (ii) can be proved similarly, so that we omit it here.

The next proposition is an analog of Proposition 3.3.

Proposition 5.9. Let $\mathfrak{A}$ be a non-empty class of metric spaces. The following conditions are equivalent.

(i) Every $X \in \mathfrak{A}$ is unshifted and every two distinct $Y, Z \in \mathfrak{A}$ are incomparable.

(ii) $\mathfrak{A}$ is minimal universal for itself.

(iii) There is a class $\mathfrak{M}$ of metric spaces such that $\mathfrak{A}$ is minimal $\mathfrak{M}$-universal.

Proof. Let (i) hold. We prove that $\mathfrak{A}$ is minimal $\mathfrak{A}$-universal. It suffices to show that (ii*) holds with $\mathfrak{M}=\mathfrak{A}$. Suppose that for a given class $\mathfrak{B}$ of metric spaces we have

$$
\mathfrak{A} \hookrightarrow \mathfrak{B} \text { and } \mathfrak{B} \preceq \mathfrak{A} .
$$

Let $X \in \mathfrak{A}$. Since $\mathfrak{A} \hookrightarrow \mathfrak{B}$, there is $Y \in \mathfrak{B}$ such that $X \hookrightarrow Y$. The condition $\mathfrak{B} \preceq \mathfrak{A}$ implies that there is $W \in \mathfrak{A}$ satisfying the inclusion $Y \subseteq W$. The last inclusion together with $X \hookrightarrow Y$ imply $X \hookrightarrow W$. Hence $X$ and $W$ are comparable. By condition (i), every two distinct $X, W \in \mathfrak{A}$ are incomparable. Hence, $X=W$ holds. Let us consider an arbitrary $f: X \hookrightarrow Y$. Since $X$ is unshifted, the isometric embedding

$$
X \stackrel{f}{\longrightarrow} Y \stackrel{i n}{\longrightarrow} W \stackrel{i d}{\longrightarrow} X,
$$

where $i n$ is the natural inclusion of $Y$ in $W$ and $i d$ is the identity map, is an isometry. Hence in $(Y)=W$, i.e., the equality $Y=X$ holds. Consequently, $\mathfrak{A} \subseteq \mathfrak{B}$ holds. Thus we have condition (ii*) with $\mathfrak{M}=\mathfrak{A}$.

The implication (ii) $\Longrightarrow$ (iii) is evident. Let us prove (iii) $\Longrightarrow$ (i). Suppose that there is a class $\mathfrak{M}$ of metric spaces such that $\mathfrak{A}$ is minimal $\mathfrak{M}$-universal. It suffices to show that every $X \in \mathfrak{A}$ is unshifted. Suppose, contrary, that $X \in \mathfrak{A}$ is shifted. Let $X_{0} \subseteq X, X \neq X_{0}$ and $X \hookrightarrow X_{0}$. Write

$$
\mathfrak{A}^{0}:=(\mathfrak{A} \backslash\{X\}) \cup\left\{X_{0}\right\} .
$$

Since $X \hookrightarrow X_{0}$ and $\mathfrak{M} \hookrightarrow \mathfrak{A}$, we have $\mathfrak{M} \hookrightarrow \mathfrak{A}^{0}$. It is clear that $\mathfrak{A}^{0} \preceq \mathfrak{A}$. Using (5.1) with $\mathfrak{B}=\mathfrak{A}^{0}$, we obtain $\mathfrak{A} \preceq \mathfrak{A}^{0}$. Hence there is $Y \in \mathfrak{A}^{0}$ such that $X \subseteq Y$. It follows directly from (5.7), that $Y \neq X_{0}$. Thus we have $Y \in \mathfrak{A}^{0} \backslash\left\{X_{0}\right\}$, i.e. $Y \in \mathfrak{A} \backslash\{X\}$. 
Hence, $X$ and $Y$ are some distinct elements of $\mathfrak{A}$. The implication

$$
(X \subseteq Y) \Longrightarrow(X \hookrightarrow Y)
$$

and condition (iii) of Definition 5.3 show that $X=Y$. Hence $X \in \mathfrak{A}^{0}$, contrary to (5.7). The implication (iii) $\Longrightarrow$ (i) follows.

As was shown in Example 3.7, there is a family $\mathfrak{Y}$ of metric spaces and there are minimal $\mathfrak{Y}$-universal metric spaces $X$ and $Y$ such that $X \not Y$. The situation is much more satisfactory when we consider the minimal $\mathfrak{Y}$-universal classes of metric spaces: such classes are always isomorphic. For the exact formulation of this result we need the following definition.

Definition 5.10. Let $\mathfrak{A}$ and $\mathfrak{B}$ be classes of metric spaces. A map $F: \mathfrak{A} \rightarrow \mathfrak{B}$ is an isomorphism if it is bijective and $F(X) \simeq X$ holds for every $X \in \mathfrak{A}$.

We shall say that classes $\mathfrak{A}$ and $\mathfrak{B}$ of metric spaces are isomorphic and write $\mathfrak{A} \simeq \mathfrak{B}$ if there is an isomorphism $F: \mathfrak{A} \rightarrow \mathfrak{B}$.

Remark 5.11. If $\mathfrak{M}, \mathfrak{A}$ and $\mathfrak{B}$ are classes of metric spaces and $\mathfrak{A} \simeq \mathfrak{B}$, and $\mathfrak{A}$ is (minimal) $\mathfrak{M}$-universal, then $\mathfrak{B}$ is also (minimal) $\mathfrak{M}$-universal.

Remark 5.12. Let $X$ and $Y$ be metric spaces and let $\mathfrak{A}=\{X\}$ and $\mathfrak{B}=\{Y\}$. Then the classes $\mathfrak{A}$ and $\mathfrak{B}$ are isomorphic if and only if $X$ and $Y$ are isometric.

Theorem 5.13. Let $\mathfrak{M}$ be a non-empty class of metric spaces. The following statements hold.

(i) If $\mathfrak{A}$ and $\mathfrak{B}$ are minimal $\mathfrak{M}$-universal classes of metric spaces, then $\mathfrak{A} \simeq \mathfrak{B}$.

(ii) If there exists a minimal $\mathfrak{M}$-universal class $\mathfrak{A}$ of metric spaces, then there exists $\mathfrak{B} \subseteq \mathfrak{M}$ such that $\mathfrak{A} \simeq \mathfrak{B}$.

Proof. (ii) Let $\mathfrak{A}$ be a minimal $\mathfrak{M}$-universal class of metric spaces. Then there is a class $\mathfrak{M}_{\mathfrak{A}}$ of metric spaces such that

$$
\mathfrak{M}_{\mathfrak{A}} \simeq \mathfrak{M} \quad \text { and } \quad \mathfrak{M}_{\mathfrak{A}} \preceq \mathfrak{A} .
$$

Since $\mathfrak{M}_{\mathfrak{A}} \preceq \mathfrak{M}_{\mathfrak{A}}$ evidently holds, condition (ii*) implies $\mathfrak{A} \subseteq \mathfrak{M}_{\mathfrak{A}}$. To prove it we use (5.3) with $\mathfrak{M}_{\mathfrak{A}}$ instead of $\mathfrak{M}$ and $\mathfrak{B}$. Now statement (ii) follows from $\mathfrak{M}_{\mathfrak{A}} \simeq \mathfrak{M}$ and $\mathfrak{A} \subseteq \mathfrak{M}_{\mathfrak{A}}$.

(i) Let $\mathfrak{A}$ and $\mathfrak{B}$ be minimal $\mathfrak{M}$-universal classes of metric spaces. We prove that $\mathfrak{A} \simeq \mathfrak{B}$. Using statement (ii), we may suppose that

$$
\mathfrak{A} \subseteq \mathfrak{M} \quad \text { and } \quad \mathfrak{B} \subseteq \mathfrak{M} .
$$

For every $X \in \mathfrak{A}$ there are $Y$ and $Y_{X}$ such that

$$
Y \in \mathfrak{B} \quad \text { and } \quad Y_{X} \subseteq Y \quad \text { and } \quad Y_{X} \simeq X
$$

Define a class $\tilde{\mathfrak{A}}$ as $\left\{Y_{X}: X \in \mathfrak{A}\right\}$. It is clear that $\tilde{\mathfrak{A}} \simeq \mathfrak{A}$ and $\tilde{\mathfrak{A}} \preceq \mathfrak{B}$. Since $\mathfrak{A}$ is minimal $\mathfrak{M}$-universal and $\tilde{\mathfrak{A}} \simeq \mathfrak{A}$, the class $\tilde{\mathfrak{A}}$ is also minimal $\mathfrak{M}$-universal. In particular, we have $\mathfrak{M} \hookrightarrow \tilde{\mathfrak{A}}$. Using condition $\left(\right.$ ii $\left.^{*}\right)$, we obtain $\mathfrak{B} \subseteq \tilde{\mathfrak{A}}$. By Corollary 5.7, the equality $\tilde{\mathfrak{A}}=\mathfrak{B}$ follows. This equality and $\tilde{\mathfrak{A}} \simeq \mathfrak{A}$ imply $\mathfrak{B} \simeq$ $\mathfrak{A}$.

Corollary 5.14. Let $\mathfrak{M}$ be a class of metric spaces and let $\mathfrak{A}$ and $\mathfrak{B}$ be two minimal $\mathfrak{M}$-universal classes of metric spaces. Then $\mathfrak{A}$ and $\mathfrak{B}$ are isomorphic.

The following proposition is a particular case of Theorem 5.13. 
Proposition 5.15. Let $\mathfrak{M}$ be an arbitrary class of metric spaces. If there exists a metric space $X$ such that the class $\{X\}$ is minimal $\mathfrak{M}$-universal, then there exists a minimal $\mathfrak{M}$-universal metric space $Y \in \mathfrak{M}$ and $Y \simeq X$.

Proof. Let $X$ be a metric space and let the class $\mathfrak{A}=\{X\}$ be minimal $\mathfrak{M}$ universal. Theorem 5.13 implies that there exists $\mathfrak{B} \subseteq \mathfrak{M}$ such that $\mathfrak{A} \simeq \mathfrak{B}$. Since $\mathfrak{A}$ is an one-element class, $\mathfrak{A} \simeq \mathfrak{B}$ and $\mathfrak{B} \subseteq \mathfrak{M}$, there is $Y \in \mathfrak{M}$ such that $\mathfrak{B}=\{Y\}$. As was noted in Remark 5.12, from $\{Y\} \simeq\{X\}$ follows that $X \simeq Y$. Since $\mathfrak{M} \hookrightarrow \mathfrak{A}$, we have $\mathfrak{M} \hookrightarrow X$. Now from $\mathfrak{M} \hookrightarrow X$ and $X \simeq Y$ it follows that $\mathfrak{M} \hookrightarrow Y$. Using Proposition 5.9, we see that $X$ is unshifted. Hence $Y$ is also unshifted. By Theorem 3.4, $Y$ is minimal $\mathfrak{M}$-universal.

A natural question is how the minimal $\mathfrak{M}$-universal subclasses of $\mathfrak{M}$ can be described.

Proposition 5.16. Let $\mathfrak{M}$ be a class of metric spaces and let $\mathfrak{A} \subseteq \mathfrak{M}$. If $\mathfrak{M} \hookrightarrow \mathfrak{A}$ holds, then the following conditions are equivalent:

(i) $\mathfrak{A}$ is minimal $\mathfrak{M}$-universal;

(ii) All metric spaces $X \in \mathfrak{A}$ are unshifted and every two distinct $Y, Z \in \mathfrak{A}$ are incomparable.

Proof. Suppose $\mathfrak{M} \hookrightarrow \mathfrak{A}$ holds. It is clear that

$$
(\mathfrak{A} \subseteq \mathfrak{M}) \Longrightarrow(\mathfrak{A} \hookrightarrow \mathfrak{M})
$$

Consequently, by Proposition 5.4, $\mathfrak{A}$ is minimal $\mathfrak{M}$-universal if and only if $\mathfrak{A}$ is minimal $\mathfrak{A}$-universal. Now the logical equivalence (i) $\Longleftrightarrow$ (ii) follows from Proposition 5.9.

We shall give a description of minimal $\mathfrak{M}$-universal subclasses $\mathfrak{A}$ of $\mathfrak{M}$ in the case when $\mathfrak{M}$ is a set of metric spaces.

Definition 5.17. A binary relation $\leq$ on a set $P$ is said to be a quasi-order if $\leq$ is reflexive and transitive. An antisymmetric quasi-order $\leq$ on $P$ is a partial order on $P$. Recall that a binary relation $\leq$ on $P$ is antisymmetric if

$$
(x \leq y \wedge y \leq x) \Longrightarrow(x=y)
$$

holds for all $x, y \in P$.

Lemma 5.18. If $\leq$ is a quasi-order on a set $P$, then a relation $\Theta_{\leq}$defined by

$$
\left(a \Theta_{\leq} b\right) \Longleftrightarrow(a \leq b \wedge b \leq a)
$$

is an equivalence relation on $P$. Moreover, a relation $\sqsubseteq$ defined on the quotient set $P / \Theta_{\leq}$as

$$
\left([a]_{\Theta_{\leq}} \sqsubseteq[b]_{\Theta_{\leq}}\right) \Longleftrightarrow\left(\exists x \in[a]_{\Theta_{\leq}} \wedge \exists y \in[b]_{\Theta_{\leq}}: x \leq y\right)
$$

is a well-defined partial order on $P / \Theta_{\leq}$.

This lemma is a standard fact from the theory of ordered sets (see, for example, $[9$, p. 141]), so that we omit the proof here.

If $X, Y$ and $Z$ are metric spaces, then we evidently have $X \hookrightarrow X$ and

$$
(X \hookrightarrow Y) \wedge(Y \hookrightarrow Z) \Longrightarrow(X \hookrightarrow Z)
$$

Hence, if $\mathfrak{M}$ is an arbitrary non-empty set of metric spaces, then the restriction of the binary relation $\hookrightarrow$ on the set $\mathfrak{M} \times \mathfrak{M}$ is an quasi-order on $\mathfrak{M}$. Let us define the 
relations $\Theta_{\hookrightarrow}$ and $\sqsubseteq$ as in (5.8) and (5.9) respectively. The following proposition is a reformulation of Lemma 5.18 for $\leq=\hookrightarrow$.

Proposition 5.19. Let $\mathfrak{M}$ be an arbitrary non-empty set of metric spaces. Then $\mathfrak{M} / \Theta_{\hookrightarrow}$ is a poset with the partial order $\sqsubseteq$.

Remark 5.20. If $X$ and $Y$ are isometric metric spaces, then the statements $X \hookrightarrow Y$ and $Y \hookrightarrow X$ are evident. In general, the converse is not true. Indeed, if $X=[0, \infty)$ and $Y=(0, \infty)$, then we have $X \hookrightarrow Y$ and $Y \hookrightarrow X$, and $X \not Y$.

Lemma 5.21. Let $X$ and $Y$ be metric spaces. Suppose $X$ is unshifted. If there exist $f: X \hookrightarrow Y$ and $g: Y \hookrightarrow X$, then $f$ and $g$ are isometries.

Proof. Since $X$ is unshifted, the mapping

$$
X \stackrel{f}{\hookrightarrow} Y \stackrel{g}{\hookrightarrow} X
$$

is an isometry. Hence $f$ and $g$ are surjections. The surjective isometric embeddings are isometries.

Lemma 5.21 implies the following corollary.

Corollary 5.22. Let $\mathfrak{M}$ be an arbitrary non-empty set of metric spaces and let $X, Y \in \mathfrak{M}$. If

$$
Y \in[X]_{\Theta \hookrightarrow}
$$

and $X$ is unshifted, then $Y$ is unshifted and $X \simeq Y$.

Let $P$ be a partially ordered set with partial order $\leq$. A point $p \in P$ is maximal if the implication

$$
(p \leq x) \Longrightarrow(x=p)
$$

holds for every $x \in P$. We shall denote by $M a x_{\leq}$the set of maximal points of the partially ordered set $P$.

Let $\mathfrak{F}=\left\{A_{i}: i \in I\right\}$ be a family of sets. A system of distinct representatives for $\mathfrak{F}$ is a set $\left\{a_{i}: i \in I\right\}$ such that $a_{i} \in A_{i}$ and $a_{i} \neq a_{j}$ for all distinct $i, j \in I$. The Axiom of Choice states that a system distinct representatives exists for each family $\left\{A_{i}: i \in I\right\}$ with mutually disjoint non-empty $A_{i}$.

Theorem 5.23. Let $\mathfrak{M}$ be an arbitrary non-empty set of metric spaces and let Max $_{\sqsubseteq}$ be the set of maximal elements of the partially ordered set $\mathfrak{M} / \Theta_{\hookrightarrow}$. The following statements are equivalent.

(i) There is a minimal $\mathfrak{M}$-universal class of metric spaces.

(ii) A system of the distinct representatives for the set $M a x_{\sqsubseteq}$ is a minimal $\mathfrak{M}$ universal class.

(iii) For every $\gamma \in \mathfrak{M} / \Theta_{\hookrightarrow}$ there are $\beta \in M a x_{\sqsubseteq}$ and $X \in \beta$ such that $\gamma \sqsubseteq \beta$ and $X$ is unshifted.

Remark 5.24. Statement (ii) of Theorem 5.23 is logically equivalent to the conjunction of the following two conditions.

(ii $\left.i_{1}\right)$ For every $\gamma \in \mathfrak{M} / \Theta_{\hookrightarrow}$ there is $\beta \in M a x_{\sqsubseteq}$ such that $\gamma \sqsubseteq \beta$.

(iii $)$ If $\gamma \in \operatorname{Max}_{\sqsubseteq}$ and $X \in \gamma$, then $X$ is unshifted.

To see this, use Corollary 5.22 and note that the implication

$$
(\beta \sqsubseteq \gamma) \Longrightarrow(\gamma=\beta)
$$

holds for all $\gamma \in \mathfrak{M} / \Theta_{\hookrightarrow}$ and $\beta \in \operatorname{Max}_{\sqsubseteq}$. 
Proof of Theorem 5.23. (i) $\Longrightarrow$ (iii). Let $\mathfrak{A}$ be a minimal $\mathfrak{M}$-universal class of metric spaces. We must show that, for every $\gamma \in \mathfrak{M} / \Theta_{\hookrightarrow}$, there are $\beta \in \operatorname{Max}_{\sqsubseteq}$ and $X \in \beta$ such that $\gamma \sqsubseteq \beta$ and $X$ is unshifted. Let $\gamma \in \mathfrak{M} / \Theta_{\hookrightarrow}$ and let $Z$ be an arbitrary metric space belonging to $\gamma$. Since $\gamma \subseteq \mathfrak{M}$ and $\mathfrak{A}$ is minimal $\mathfrak{M}$-universal, then there is $Y \in \mathfrak{A}$ such that $Z \hookrightarrow Y$. By Theorem 5.13, there is $X \in \mathfrak{M}$ such that $X \simeq Y$. Consequently, $Z \hookrightarrow X$. Define $\beta:=[X]_{\Theta_{\hookrightarrow}}$. Using (5.9) with $a=Z, b=X$ and $\Theta_{<}=\Theta_{\hookrightarrow}$, we obtain from $Z \hookrightarrow X$ that $\gamma \sqsubseteq \beta$. To prove that $X$ is unshifted recall that $X \simeq Y$ and $Y \in \mathfrak{A}$ where $\mathfrak{A}$ is minimal $\mathfrak{M}$-universal. By Proposition 5.9, the space $Y$ is unshifted. Hence $X$ is unshifted as a space which is isometric to $Y$. It still remains to prove that $\beta \in \operatorname{Max}_{\sqsubseteq}$. The statement $\beta \in \operatorname{Max}_{\sqsubseteq}$ holds if for every $\alpha \in \mathfrak{M} / \Theta_{\hookrightarrow}$ the inequality $\beta \sqsubseteq \alpha$ implies $\beta=\alpha$. Let $\beta=[X]_{\Theta_{\hookrightarrow}}$ and $\alpha=[W]_{\Theta \hookrightarrow}$ where $X$ is the same as above and $W$ is an arbitrary element of $\alpha$. Inequality $\beta \sqsubseteq \alpha$ implies that $X \hookrightarrow W$. Since $\alpha \subseteq \mathfrak{M}$, we have $W \in \mathfrak{M}$. Consequently, there is a space $Q \in \mathfrak{A}$ such that $W \hookrightarrow Q$. Let $X \hookrightarrow Y$ with the same $Y \in \mathfrak{A}$ as above. By Proposition 5.9, we have either $Q=Y$ or $Q$ and $Y$ are incomparable. Since $X \hookrightarrow W, W \hookrightarrow Q$ and $Y \simeq X$ hold, the metric spaces $Q$ and $Y$ are comparable. Hence, the equality $Q=Y$ holds. In particular, we obtain $Q \hookrightarrow X$. From $X \hookrightarrow W$, $W \hookrightarrow Q$ and $Q \hookrightarrow X$ it follows that $X \hookrightarrow W$ and $W \hookrightarrow X$. Hence we have $\beta=[X]_{\Theta_{\hookrightarrow}}=[W]_{\Theta_{\hookrightarrow}}=\alpha$. The implication (i) $\Longrightarrow$ (iii) follows.

(iii) $\Longrightarrow$ (ii). Suppose condition (iii) holds. Let

$$
\mathfrak{A}=\left\{A_{\alpha}: \alpha \in \operatorname{Max}_{\sqsubseteq}\right\}
$$

where $A_{\alpha} \in \alpha$, be a system of distinct representatives for the set $M a x_{\sqsubseteq}$. We claim that $\mathfrak{A}$ is minimal $\mathfrak{M}$-universal. We first establish that $\mathfrak{A}$ is $\mathfrak{M}$-universal. Let $X \in \mathfrak{M}$ and let $\gamma \in \mathfrak{M} / \Theta_{\hookrightarrow}$ such that $X \in \gamma$. By (iii), there is $\beta \in \operatorname{Max}_{\sqsubseteq}$ such that $\gamma \sqsubseteq \beta$. Using (5.9), we obtain $X \hookrightarrow A_{\beta}$. Using (5.9) it is also easy to prove that $A_{\alpha}$ and $A_{\beta}$ are incomparable if $\alpha \neq \beta$. Hence, conditions (i) and (iii) of Definition 5.3 are satisfied. We end the proof by demonstration that condition (ii) of this definition holds. In accordance with Remark 5.24, we can suppose that all $A_{\alpha}$ are unshifted. Hence, by Proposition 5.9, $\mathfrak{A}$ is minimal universal for $\mathfrak{A}$ itself. Consequently, by Definition 5.3, we have

$$
(\mathfrak{A} \hookrightarrow \mathfrak{B}) \wedge(\mathfrak{B} \preceq \mathfrak{A}) \Longrightarrow(\mathfrak{A} \preceq \mathfrak{B})
$$

for every class $\mathfrak{B}$ of metric spaces. Since $\mathfrak{A} \preceq \mathfrak{M}$, we have

$$
(\mathfrak{M} \hookrightarrow \mathfrak{B}) \wedge(\mathfrak{B} \preceq \mathfrak{A}) \Longrightarrow(\mathfrak{A} \hookrightarrow \mathfrak{B}) \wedge(\mathfrak{B} \preceq \mathfrak{A}) .
$$

Consequently, we obtain (5.1),

$$
(\mathfrak{M} \hookrightarrow \mathfrak{B}) \wedge(\mathfrak{B} \preceq \mathfrak{A}) \Longrightarrow(\mathfrak{A} \preceq \mathfrak{B})
$$

for every class $\mathfrak{B}$ of metric spaces. Condition (ii) of Definition 5.3 follows.

To complete the proof, it suffices to observe that the implication (ii) $\Longrightarrow$ (i) is trivial.

We finish the section by constructing a minimal universal class for the class of linear normed spaces of given finite dimension.

Lemma 5.25. Let $X$ be a complete non-empty metric space and let $Y$ be a connected metric space. Then every open $f: X \hookrightarrow Y$ is an isometry.

Proof. Let $f: X \hookrightarrow Y$ be open. Then $f(X)$ is open and complete. This implies that $f(X)$ is clopen in the connected space $Y$. Hence, $f(X)=Y$ holds. 
Proposition 5.26. Let $n$ be a positive integer number and let $\mathcal{N}_{n}$ be the class of all normed $n$-dimensional linear spaces over the field $\mathbf{R}$. Then there is a minimal $\mathcal{N}_{n}$-universal subset of $\mathcal{N}_{n}$.

Proof. Every finite-dimensional normed linear space over $\mathbf{R}$ is separable. Hence, by the Banach-Mazur theorem, for every $Y \in \mathcal{N}_{n}$, there is $Y^{\prime} \subseteq C[0,1]$ such that $Y \simeq Y^{\prime}$. Consequently, without loss of generality, we may identify $\mathcal{N}_{n}$ with a subset of $2^{C[0,1]}$ and denote this subset by the same symbol $\mathcal{N}_{n}$. Let $\left\{[Y]: Y \in \mathcal{N}_{n}\right\}$ be the quotient set of $\mathcal{N}_{n}$ by the relation $\simeq$, where, as above, $X \simeq Y$ means that $X$ and $Y$ are isometric. Let $\mathfrak{M}$ be a system of representatives for $\left\{[Y]: Y \in \mathcal{N}_{n}\right\}$. It is easy to see that $\mathfrak{M} \subseteq \mathcal{N}_{n}$ and $\mathcal{N}_{n} \hookrightarrow \mathfrak{M}$. We claim that $\mathfrak{M}$ is a minimal $\mathcal{N}_{n}$-universal set of metric spaces. By Proposition 5.16, $\mathfrak{M}$ is minimal $\mathcal{N}_{n}$-universal if and only if:

$\left(\mathrm{i}_{1}\right)$ Every $Y \in \mathfrak{M}$ is unshifted;

$\left(\mathrm{i}_{2}\right)$ Every two distinct $X, Y \in \mathfrak{M}$ are incomparable.

Since every finite-dimensional normed linear space over $\mathbf{R}$ is homogeneous and boundedly compact, property $\left(i_{1}\right)$ follows from Lemma 3.23. Let us consider $\left(i_{2}\right)$. Brouwer's invariance of domain theorem says that a continuous injective map $f: U \rightarrow \mathbf{R}^{n}$ is open for every open $U \subseteq \mathbf{R}^{n}$ (see, for example, [51, p. 34]). Since all $X, Y \in \mathfrak{M}$ are homeomorphic to $\mathbf{R}^{n}$, we see that conditions of Lemma 5.25 are valid for every $f: X \hookrightarrow Y$ with $X, Y \in \mathfrak{M}$. From this lemma and from the definition of $f$ it follows that if $X$ and $Y$ are comparable and $X, Y \in \mathfrak{M}$, then $X=Y$. Condition $\left(\mathrm{i}_{2}\right)$ follows.

Remark 5.27. Using the minimal $\mathcal{N}_{n}$-universal set $\mathfrak{M}$ that was constructed in the proof of Proposition 5.26, we can construct a disjoint union

$$
X=\coprod_{Y \in \mathfrak{M}} Y
$$

such that $X$ is a minimal $\mathcal{N}_{n}$-universal metric space. See Corollary 6.13 in the next section.

\section{From minimal universal classes to minimal universal spaces. Disjoint unions of metric spaces}

In this section we shall denote by $I$ a non-empty index set.

Definition 6.1. A metric space $X$ is a disjoint union of metric spaces $Y_{i}, i \in I$, if there is a partition $\left\{X_{i}: i \in I\right\}$ of the set $X$ such that $X_{i} \simeq Y_{i}$ for every $i \in I$. In this case we write

$$
X=\coprod_{i \in I} Y_{i}
$$

Recall that $\left\{X_{i}: i \in I\right\}$ is a partition of $X$ if $X=\bigcup_{i \in I} X_{i}, X_{i} \neq \varnothing$ and $X_{i} \cap X_{j}=$ $\varnothing$ for all distinct $i, j \in I$. In particular, if $X=\coprod_{i \in I} Y_{i}$, then all spaces $Y_{i}$ are nonempty.

The next proposition follows from Proposition 3.8 of [13].

Proposition 6.2. A disjoint union $\coprod_{i \in I} Y_{i}$ exists for every family of non-empty metric spaces $Y_{i}$.

It is clear that $\mathfrak{M} \hookrightarrow \coprod_{i \in I} Y_{i}$ holds for $\mathfrak{M}=\left\{Y_{i}: i \in I\right\}$. A legitimate question to raise at this point is whether there exists a metric $d_{X}$ on $X=\coprod_{i \in I} Y_{i}$ such that $X$ is minimal $\mathfrak{M}$-universal. 
Proposition 6.3. Let $\mathfrak{M}=\left\{Y_{i}: i \in I\right\}$ be a set of metric spaces. If a disjoint union $X=\coprod_{i \in I} Y_{i}$ is a minimal $\mathfrak{M}$-universal metric space, then all $Y_{i} \in \mathfrak{M}$ are unshifted and pairwise incomparable.

Proof. For simplicity, we assume that the metric spaces $Y_{i}, i \in I$, are pairwise disjoint, $Y_{i} \cap Y_{j}=\varnothing$ if $i \neq j$, and $X=\bigcup_{i \in I} Y_{i}$ such that the restriction $\left.d_{X}\right|_{Y_{i} \times Y_{i}}$ is equal to $d_{Y_{i}}$ for every $i \in I$.

Let $X$ be minimal $\mathfrak{M}$-universal. Suppose that, contrary to our claim, $Y_{i_{1}} \hookrightarrow Y_{i_{2}}$ for some distinct $i_{1}, i_{2} \in I$. Then the metric space

$$
X^{i_{1}}=\coprod_{i \in I, i \neq i_{1}} Y_{i}
$$

with the metric induced from $X$, is $\mathfrak{M}$-universal and $X \backslash X^{i_{1}} \neq \varnothing$, in contradiction with the minimal universality of $X$.

Similarly, if $Y_{i_{1}}$ is shifted and $Y_{i_{1}}^{0} \subseteq Y_{i_{1}}$ such that

$$
Y_{i_{1}} \simeq Y_{i_{1}}^{0} \text { and } Y_{i_{1}} \backslash Y_{i_{1}}^{0} \neq \varnothing
$$

then the metric space

$$
X_{0}^{i_{1}}=\left(\coprod_{i \in I, i \neq i_{1}} Y_{i}\right) \sqcup Y_{i_{1}}^{0}
$$

with the metric induced from $X$, is $\mathfrak{M}$-universal, contrary to the condition.

Remark 6.4. In the next section (see Corollary 7.6), we shall construct a family $\mathfrak{M}=\left\{Y_{i}: i \in I\right\}$ such that:

- $\mathfrak{M}$ admits a minimal universal metric space;

- Every $Y_{i}$ is unshifted and every two distinct $Y_{i}, Y_{j}$ are incomparable;

- There is no minimal $\mathfrak{M}$-universal $X$ having a form $X=\coprod_{i \in I} Y_{i}$.

Proposition 6.3 and Proposition 5.9 imply the following.

Corollary 6.5. If $X=\coprod_{i \in I} Y_{i}$ is a minimal universal metric space for a class $\mathfrak{M}=\left\{Y_{i}: i \in I\right\}$, then there is a class $\mathfrak{A}$ of metric spaces such that $\mathfrak{M}$ is minimal $\mathfrak{A}$-universal.

The following theorem describes, for given family $\mathfrak{M}$ of metric spaces, the structure of minimal $\mathfrak{M}$-universal metric spaces $X$ which have a form $X=\coprod_{Y \in \mathfrak{M}} Y$.

Theorem 6.6. Let $\mathfrak{M}=\left\{Y_{i}: i \in I\right\}$ be a set of non-empty metric spaces and let $X$ be a minimal $\mathfrak{M}$-universal metric space. Suppose that all $Y_{i} \in \mathfrak{M}$ are unshifted and pairwise incomparable. Then the following statements are equivalent.

(i) The metric space $X$ is a disjoint union of metric spaces $Y_{i}, i \in I$,

$$
X=\coprod_{i \in I} Y_{i}
$$

(ii) The metric space $X$ satisfies the following conditions.

(ii $\left.i_{1}\right)$ For every $x_{0} \in X$, there is a unique $Y_{i_{0}} \in \mathfrak{M}$ such that

$$
Y_{i_{0}} \nrightarrow X \backslash\left\{x_{0}\right\} .
$$

(ii ${ }_{2}$ ) For every non-empty subset $\mathfrak{M}_{0}$ of the set $\mathfrak{M}$, there is a unique minimal $\mathfrak{M}_{0}$-universal metric subspace $X^{0}$ of the space $X$. 
Proof. Let (i) hold. Then, by Definition 6.1, there is a partition $\left\{X_{i}: i \in I\right\}$ of $X$ such that $X_{i} \simeq Y_{i}$ holds for every $i \in I$. If $x_{0}$ is an arbitrary point of $X$, then there is a unique $i_{0} \in I$ with $x_{0} \in X_{i_{0}}$. It is clear that

$$
Y_{i} \hookrightarrow \bigcup_{i \in I, i \neq i_{0}} X_{i} \hookrightarrow X \backslash\left\{x_{0}\right\}
$$

holds for every $i \in I \backslash\left\{i_{0}\right\}$. Consequently we have

$$
Y_{i_{0}} \nrightarrow X \backslash\left\{x_{0}\right\}
$$

because $X$ is minimal $\mathfrak{M}$-universal. The implication $(\mathrm{i}) \Longrightarrow\left(\mathrm{ii}_{1}\right)$ follows.

To deal with $(\mathrm{i}) \Longrightarrow\left(\mathrm{ii}_{2}\right)$, consider an arbitrary non-empty $I_{0} \subseteq I$. Let $\mathfrak{M}_{0}=\left\{Y_{i}: i \in I_{0}\right\}$. It is easy to see that the metric space $\bigcup_{i \in I_{0}} X_{i}$, with the metric induced from $X$, is minimal $\mathfrak{M}_{0}$-universal. Indeed, it is clear that $\mathfrak{M}_{0} \hookrightarrow \bigcup_{i \in I_{0}} X_{i}$. If there is $X^{0} \subseteq \bigcup_{i \in I_{0}} X_{i}$ such that $\mathfrak{M}_{0} \hookrightarrow X^{0}$ and

$$
\left(\bigcup_{i \in I_{0}} X_{i}\right) \backslash X^{0} \neq \varnothing
$$

then

$$
\mathfrak{M} \hookrightarrow X^{0} \cup\left(\bigcup_{i \in I \backslash I_{0}} X_{i}\right)
$$

because

$$
\mathfrak{M}=\mathfrak{M}_{0} \cup\left(\mathfrak{M} \backslash \mathfrak{M}_{0}\right) \quad \text { and } \quad \mathfrak{M} \backslash \mathfrak{M}_{0} \hookrightarrow \bigcup_{i \in I \backslash I_{0}} X_{i}
$$

It follows from (6.1) and (6.2) that $X$ is not minimal $\mathfrak{M}$-universal, contrary to the condition of the theorem. Now suppose that $X^{1} \subseteq X$ is minimal $\mathfrak{M}_{0}$-universal and

$$
X^{1} \neq \bigcup_{i \in I_{0}} X_{i}
$$

If $X^{1} \supseteq \bigcup_{i \in I_{0}} X_{i}$, then (6.3) implies that $X^{1}$ is not minimal $\mathfrak{M}_{0}$-universal. Hence, there is $x_{0} \in \bigcup_{i \in I_{0}} X_{i}$ such that $x_{0} \notin X^{1}$. Consequently, we have

$$
\mathfrak{M} \hookrightarrow X^{1} \cup\left(\bigcup_{i \in I \backslash I_{0}} X_{i}\right) \quad \text { and } \quad X^{1} \cup\left(\bigcup_{i \in I \backslash I_{0}} X_{i}\right) \subseteq X \backslash\left\{x_{0}\right\},
$$

contrary to the minimality of $X$. The implication $(\mathrm{i}) \Longrightarrow\left(\mathrm{ii}_{2}\right)$ is proved, so that (i) $\Longrightarrow$ (ii) follows.

Let us prove the validity of (ii) $\Longrightarrow$ (i). Suppose that (ii) holds. Since $X$ is $\mathfrak{M}$-universal, for every $i \in I$, there is $f_{i}: Y_{i} \hookrightarrow X$. Write $X_{i}=f_{i}\left(Y_{i}\right)$. It is clear that $X_{i} \simeq Y_{i}$ holds for every $i \in I$. Hence it suffices to prove that $\left\{X_{i}: i \in I\right\}$ is a partition of $X$. All sets $Y_{i}$ are non-empty. Hence all $X_{i}$ are non-empty also. Since $X$ is minimal $\mathfrak{M}$-universal and

$$
\mathfrak{M} \hookrightarrow \bigcup_{i \in I} X_{i} \text { and } \bigcup_{i \in I} X_{i} \subseteq X
$$

we have $X=\bigcup_{i \in I} X_{i}$. It remains to prove that

$$
X_{i} \cap X_{j}=\varnothing
$$


holds for every pair distinct $i, j \in I$. Suppose contrary that there exist $i_{1}, i_{2} \in I$ with

$$
X_{i_{1}} \cap X_{i_{2}} \neq \varnothing \quad \text { and } \quad i_{1} \neq i_{2} .
$$

Let $i \in I$. Since $Y_{i} \in \mathfrak{M}$ is unshifted, Proposition 3.3 implies, for every $A_{i} \subseteq X$ with $A_{i} \simeq Y_{i}$, that $A_{i}$ is a minimal universal metric space for the class $\left\{Y_{i}\right\}$. Using condition $\left(\mathrm{ii}_{2}\right)$, with $\mathfrak{M}_{0}=\left\{Y_{i_{k}}\right\}, k=1,2$, we obtain that there is a unique $A_{i_{k}} \subseteq X$ such that $A_{i_{k}} \simeq Y_{i_{k}}$. By definition, $X_{i_{k}} \subseteq X$ and, moreover, $X_{i_{k}} \simeq Y_{i_{k}}$ holds for $k=1,2$. Consequently, if

$$
x_{0} \in X_{i_{1}} \cap X_{i_{2}}
$$

then we have

$$
Y_{i_{1}} \nrightarrow X \backslash\left\{x_{0}\right\} \text { and } Y_{i_{2}} \nrightarrow X \backslash\left\{x_{0}\right\}
$$

contrary to condition $\left(\mathrm{ii}_{2}\right)$.

Remark 6.7. Theorem 6.6 remains valid if we replace condition $\left(\mathrm{ii}_{2}\right)$ by the following:

$\left(\mathrm{ii}_{2}^{0}\right)$ For every $Y_{i} \in \mathfrak{M}$, there is a unique $X^{i} \subseteq X$ such that $X^{i} \simeq Y_{i}$.

Proof. By the hypothesis of Theorem 6.6, all $Y_{i} \in \mathfrak{M}$ are unshifted. From Proposition 3.3 it follows that a metric space $X^{i}$ is minimal universal for the class $\left\{Y_{i}\right\}$ if and only if $X^{i} \simeq Y_{i}$. Hence $\left(\mathrm{ii}_{2}^{0}\right)$ is an equivalent for the condition:

$\left(\mathrm{ii}_{2}^{1}\right)$ For every $i \in I$ there is a unique $X^{i} \subseteq X$ which is minimal universal for $\left\{Y_{i}\right\}$. We evidently have $\left(\mathrm{ii}_{2}\right) \Longrightarrow\left(\mathrm{ii}_{2}^{1}\right)$. To complete the proof it suffices to note that in the proof of the implication (ii) $\Longrightarrow$ (i) we use, in fact, condition ( $\mathrm{ii}_{2}^{1}$ ) instead of $\left(\mathrm{ii}_{2}\right)$.

Theorem 6.8. Let $\mathfrak{M}=\left\{Y_{i}: i \in I\right\}$ be a family of non-empty metric spaces and let $X=\coprod_{i \in I} Y_{i}$. Then $X$ is a minimal $\mathfrak{M}$-universal metric space if and only if the following statements hold.

(i) Every $Y_{i} \in \mathfrak{M}$ is unshifted.

(ii) Metric spaces $Y_{i}$ and $Y_{j}$ are incomparable for distinct $i, j \in I$.

(iii) For every $Y_{i} \in \mathfrak{M}$ there is a unique $X^{i} \subseteq X$ such that $Y_{i} \simeq X^{i}$.

Proof. Suppose that $X$ is minimal $\mathfrak{M}$-universal. Then, by Proposition 6.3 , conditions (i) and (ii) are valid. Now Theorem 6.6 implies (iii). Conversely, if (i), (ii) and (iii) hold, then, using Theorem 6.6 and Remark 6.7, we see that $X$ is minimal $\mathfrak{M}$-universal if and only if condition ( $\mathrm{ii}_{1}$ ) of Theorem 6.6 holds. To prove (ii ${ }_{1}$ ) suppose that $x_{0}$ is an arbitrary point of $X$. Since we have $X=\coprod_{i \in I} Y_{i}$, there is a partition $\left\{X_{i}: i \in I\right\}$ of $X$ such that $X_{i} \simeq Y_{i}$ for every $i \in I$. Let $X_{i_{0}}$ be a part such that $x_{0} \in X_{i_{0}}, i_{0} \in I$. It suffices to show that $Y_{i_{0}} \triangleleft X_{X} \backslash\left\{x_{0}\right\}$ and

$$
\left(Y_{i} \nrightarrow X \backslash\left\{x_{0}\right\}\right) \Longrightarrow\left(i=i_{0}\right)
$$

for every $i \in I$. As in the proof of Theorem 6.6, we obtain $Y_{i} \hookrightarrow X \backslash\left\{x_{0}\right\}$ for $i \in I \backslash\left\{i_{0}\right\}$. Suppose we also have $Y_{i_{0}} \hookrightarrow X \backslash\left\{x_{0}\right\}$. Then there is $X^{i_{0}} \subseteq X \backslash\left\{x_{0}\right\}$ such that $Y_{i_{0}} \simeq X^{i_{0}}$. Thus we obtain $X^{i_{0}} \neq X_{i_{0}}$, because $x_{0} \notin X^{i_{0}}$ and $x_{0} \in X_{i_{0}}$. This is a contradiction with (iii).

Let us give some applications of Theorem 6.8 to construction of minimal universal metric spaces based on the disjoint unions. 
Definition 6.9. Let $\varepsilon \in(0, \infty)$. A metric space $X$ is said to be $\varepsilon$-connected if for every $x, y \in X$ there is a finite sequence $\left(x_{i}\right), i=1, \ldots, n$, such that: $x_{i} \in X$, for all $i \in 1, \ldots, n$ and $x_{1}=x, x_{n}=y$ and

$$
d_{X}\left(x_{i}, x_{i+1}\right) \leq \varepsilon
$$

for every $i \in\{1, \ldots, n-1\}$.

Theorem 6.10. Let $\mathfrak{M}=\left\{Y_{i}: i \in I\right\}$ be a set of non-empty metric spaces. Suppose all $Y_{i}$ are unshifted and pairwise incomparable. If there exists $\varepsilon>0$ such that $Y_{i}$ is $\varepsilon$-connected for every $i \in I$, then there is a metric on the disjoint union

$$
X=\coprod_{i \in I} Y_{i}
$$

such that $X$ with this metric is a minimal $\mathfrak{M}$-universal metric space.

The proof of Theorem 6.10 can be divided into the following three steps.

- We consider disjoint metric spaces $X_{i} \simeq Y_{i}, i \in I$ and construct a connected weighted graph $(G, w)$ with the vertex set $V(G)$, the edge set $E(G)$ and the weight $w: E(G) \rightarrow \mathbf{R}^{+}, \mathbf{R}^{+}=[0, \infty)$, such that:

$-V(G)=X$ with $X=\bigcup_{i \in I} X_{i}$;

- If $x, y$ are distinct points of $X_{i}, i \in I$, then $\{x, y\} \in E(G)$;

- The equality

$$
w(\{x, y\})=d_{X_{i}}(x, y)
$$

holds for every $i \in I$ and all distinct $x, y \in X_{i}$.

- Using some results of [13], we show that the weighted shortest path pseudometric $d_{w, X}$ is a metric on $X$ satisfying the equality

$$
d_{w, X}(x, y)=d_{X_{i}}(x, y)
$$

for every $i \in I$ and all $x, y \in X_{i}$.

- We apply Theorem 6.8 to prove that $\left(X, d_{w, X}\right)$ is minimal $\mathfrak{M}$-universal.

In the proof of Theorem 6.10 we will use a basic terminology of the Graph Theory (see, for example, [6]). All our graphs $G$ are simple, so that we can identify the edge set $E(G)$ with a set of two-elements subsets of the vertex set $V(G)$. We use the standard definition of paths and cycles. It should be noted that all vertices of any cycle $C$ can be labeled as $x_{0}, \ldots, x_{n}$ with $|V(C)|=n, x_{0}=x_{n}$ and, for $0 \leqslant i<j \leqslant n$, $\left\{x_{j}, x_{i}\right\} \in E(C)$ if and only if $j-i=1$.

Recall that a symmetric function $\rho: X \times X \rightarrow \mathbf{R}^{+}$is a pseudometric on $X$ if $\rho$ satisfies the triangle inequality $\rho(x, y) \leq \rho(x, z)+\rho(z, y)$ and the equality $\rho(x, x)=0$ for all $x, y, z \in X$, but, in general, the implication

$$
(\rho(x, y)=0) \Longrightarrow(x=y)
$$

need not hold.

Definition 6.11. Let $(G, w)$ be a weighted graph with $E(G) \neq \varnothing$. The weight $w$ is pseudometrizable if there is a pseudometric $d_{V}$ on the vertex set $V=V(G)$ such that

$$
d_{V}(x, y)=w(\{x, y\}) \text { for every }\{x, y\} \in E(G) .
$$

A pseudometrizable weight $w$ is metrizable if there is a metric $d_{V}$ satisfying (6.4). 
Let $(G, w)$ be a weighted graph and let $u, v$ be distinct vertices of $G$. Let us denote by $P_{u, v}$ the set of all paths joining $u$ and $v$ in $G$. Thus, a finite sequence $\left(t_{0}, \ldots, t_{n}\right)$ belongs to $P_{u, v}$ if and only if $u=t_{0}, v=t_{n}, t_{i} \in V(G)$ for $0 \leq i \leq n$, and $t_{i} \neq t_{j}$ for $0 \leq i<j \leq n$ and $\left\{t_{i}, t_{i+1}\right\} \in E(G)$ for $0 \leq i \leq n-1$.

Let $u, v \in V(G)$. Write

$$
d_{w, G}(u, v):= \begin{cases}\inf \left\{w(F): F \in P_{u, v}\right\} & \text { if } u \neq v, \\ 0 & \text { if } u=v .\end{cases}
$$

Recall that a graph $G$ is connected if $P_{u, v} \neq \varnothing$ for all distinct $u, v \in V(G)$. For the connected weighted graphs it is well known that the function $d_{w, G}$ is a pseudometric on the set $V(G)$. This pseudometric will be termed the weighted shortest path pseudometric. It coincides with the usual path metric if $w(e)=1$ for every $e \in E(G)$.

If $(G, w)$ is a weighted graph, then for each subgraph $F$ of the graph $G$ define

$$
w(F):=\sum_{e \in E(F)} w(e) .
$$

The next lemma follows immediately from Proposition 2.1 and Proposition 3.1 of [13].

Lemma 6.12. Let $(G, w)$ be a connected weighted graph with $V(G) \neq \varnothing$. The following conditions are equivalent:

(i) The weight $w$ is pseudometrizable;

(ii) For every cycle $C=\left(t_{0}, t_{1}, \ldots, t_{n}\right)$ in $G$ we have the inequality

$$
2 \max _{0 \leq i \leq n-1} w\left\{t_{i}, t_{i+1}\right\} \leq w(C) .
$$

Moreover, a pseudometrizable weight $w$ is metrizable if and only if the inequality

$$
\inf \left\{w(F): F \in P_{u, v}\right\}>0
$$

holds for every pair of distinct $u, v \in V(G)$. If $w$ is metrizable, then the weighted shortest path pseudometric $d_{w, G}$ is a metric and condition (6.4) holds with $d_{V}=d_{w, G}$.

Proof of Theorem 6.10. Let $\left\{X_{i}: i \in I\right\}$ be a family of metric spaces satisfying the conditions

$$
X_{i} \cap X_{j}=\varnothing \quad \text { and } \quad X_{i} \simeq Y_{i}
$$

for all distinct $i, j \in I$. Write

$$
X:=\bigcup_{i \in I} X_{i}
$$

By conditions of the theorem all $Y_{i}$ are unshifted and $Y_{i} \hookrightarrow Y_{j}$ if $i \neq j$ and $i, j \in I$. Suppose that there is $\varepsilon>0$ such that $Y_{i}$ is $\varepsilon$-connected for every $i \in I$. Since, for every $i \in I, Y_{i}$ is not empty, there is a system of distinct representatives $\left\{a_{i}: i \in I\right\}$ for the family $\left\{X_{i}: i \in I\right\}$, i.e.,

$$
a_{i} \in X_{i} \quad \text { and } \quad a_{i} \neq a_{j}
$$

for all distinct $i, j \in I$. Let us consider a graph $G$ with $V(G)=X$ and the edge set $E(G)$ defined by the rule: if $x, y$ are distinct points of $X$, then

$$
(\{x, y\} \in E(G)) \Longleftrightarrow\left(\left(\exists i \in I: x, y \in X_{i}\right) \vee\left(\exists i, j \in I: x=a_{i} \wedge y=a_{j}\right)\right)
$$

(see Figure 1). The graph $G$ is connected, because $\left\{X_{i}: i \in I\right\}$ is a partition of $X=V(G)$. 

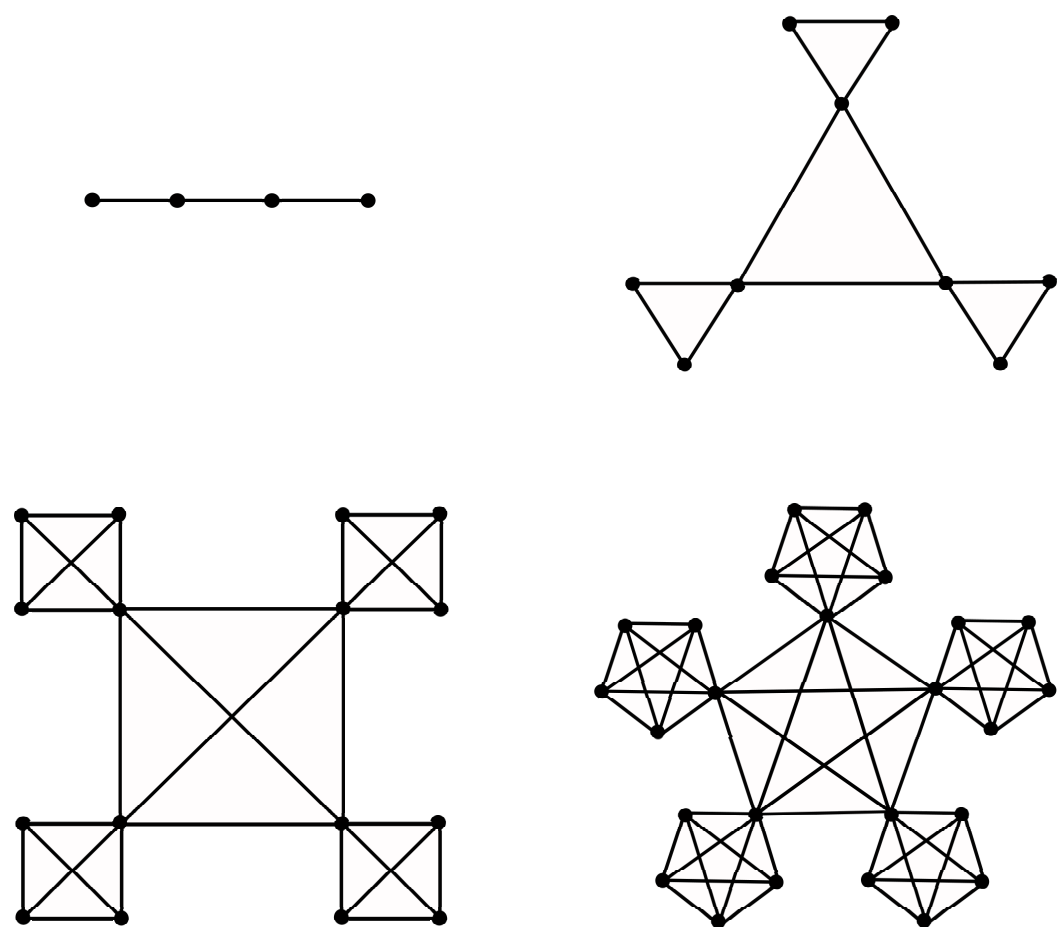

Figure 1. The graphs $G$ for the families $\left\{Y_{i}: i \in I\right\}$ with $I=\{1,2, \ldots, k\}$ and $\left|Y_{k}\right|=k$, $k=2,3,4,5$.

Let $\varepsilon<\varepsilon_{1}<\infty$. Define a weight $w: E(G) \rightarrow \mathbf{R}^{+}$as follows

$$
w(\{x, y\}):= \begin{cases}d_{X_{i}}(x, y) & \text { if }\{x, y\} \subseteq X_{i} \text { for some } i \in I, \\ \varepsilon_{1} & \text { if } x=a_{i} \text { and } y=a_{j} \text { for some distinct } i, j \in I .\end{cases}
$$

By Lemma 6.12, the weight $w$ is pseudometrizable if inequality (6.6) holds for every cycle $C$ in $G$. Let $C=\left(t_{0}, \ldots, t_{n}\right), t_{0}=t_{n}$, be an arbitrary cycle in $G$. If there is $i \in I$ such that $V(C) \subseteq X_{i}$, then (6.6) holds because $C$ is a cycle in the induced subgraph $G\left[X_{i}\right]$ for which the restriction $\left.w\right|_{E\left(G\left[X_{i}\right]\right)}$ of the weight function $w$ is evidently metrizable. Recall that, by the definition of induced subgraphs, we have $V\left(G\left[X_{i}\right]\right)=X_{i}$ and, for every $x, y \in X_{i}$

$$
\left(\{x, y\} \in E\left(G\left[X_{i}\right]\right)\right) \Longleftrightarrow(\{x, y\} \in E(G)) .
$$

Suppose now that

$$
V(C) \nsubseteq X_{i} \quad \text { for any } i \in I .
$$

Let $f: X \rightarrow I$ be a function satisfying the statement

$$
(f(x)=i) \Longleftrightarrow\left(x \in X_{i}\right)
$$

for all $x \in X, i \in I$. Condition (6.10) implies the existence of $k \in\{0, \ldots, n-1\}$ such that

$$
f\left(t_{k}\right) \neq f\left(t_{k+1}\right) \text {. }
$$

Indeed, if the equality $f\left(t_{k}\right)=f\left(t_{k+1}\right)$ holds for every $k \in\{0, \ldots, n-1\}$, then we have

$$
V(C)=\left\{t_{0}, \ldots, t_{n-1}\right\} \subseteq X_{f\left(t_{0}\right)},
$$

contrary to (6.10). After a renumbering of the vertices of $C$, we may suppose

$$
f\left(t_{0}\right) \neq f\left(t_{1}\right) .
$$


It follows from (6.12) and the definitions of $w$ and $f$ that

$$
t_{0}=a_{f\left(t_{0}\right)} \quad \text { and } \quad t_{1}=a_{f\left(t_{1}\right)} .
$$

In addition, since $t_{0}=t_{n}$, we also have

$$
t_{n}=a_{f\left(t_{n}\right)} .
$$

We claim that (6.13) and (6.14) together with (6.8) imply the equality

$$
t_{i}=a_{f\left(t_{i}\right)}
$$

for every $i \in\{0, \ldots, n\}$. Suppose contrary that there is $i \in\{0, \ldots, n\}$ such that $t_{i} \neq a_{f\left(t_{i}\right)}$. Then we can find the smallest index $i_{0}$ such that

$$
t_{i_{0}} \neq a_{f\left(t_{i_{0}}\right)} \quad \text { and } \quad t_{i}=a_{f\left(t_{i}\right)} \quad \text { for } \quad i \in\left\{0, \ldots, i_{0}-1\right\} .
$$

From the definition of $i_{0}$ and (6.14), we have

$$
t_{i_{0}-1}=a_{f\left(t_{i_{0}-1}\right)} \quad \text { with } \quad 2 \leq i_{0}-1<i_{0} \leq n-1 .
$$

Since

$$
\left\{t_{i_{0}-1}, t_{i_{0}}\right\} \in E(C) \subseteq E(G) \text { and } t_{i_{0}} \in X_{f\left(t_{i_{0}}\right)} \backslash\left\{a_{f\left(t_{i_{0}}\right)}\right\},
$$

rule (6.8) implies that

$$
f\left(t_{i_{0}}\right)=f\left(t_{i_{0}-1}\right) .
$$

Let $i_{1} \in\left\{i_{0}, i_{0}+1, \ldots, n\right\}$ such that

$$
t_{i_{0}} \neq a_{f\left(t_{i_{0}}\right)}, t_{i_{0}+1} \neq a_{f\left(t_{i_{0}+1}\right)}, \ldots, t_{i_{1}} \neq a_{f\left(t_{i_{1}}\right)}
$$

and

$$
t_{i_{1}+1}=a_{f\left(t_{i_{1}+1}\right)} .
$$

Then we have $i_{0} \leq i_{1} \leq n-1$ and, similarly (6.17), the equality

$$
f\left(t_{i_{1}}\right)=f\left(t_{i_{1}+1}\right)
$$

holds. Using (6.18) and (6.8) we can also prove the chain of the equalities

$$
f\left(t_{i_{0}}\right)=f\left(t_{i_{0}+1}\right)=\ldots=f\left(t_{i_{1}+1}\right) .
$$

Thus we have $t_{i_{0}-1}=a_{f\left(t_{i_{0}-1}\right)}$, by (6.16), $a_{f\left(t_{i_{0}-1}\right)}=a_{f\left(t_{i_{0}}\right)}$, by (6.17),

$$
a_{f\left(t_{i_{0}}\right)}=a_{f\left(t_{i_{0}+1}\right)}=\ldots=a_{f\left(t_{i_{1}+1}\right)},
$$

by $(6.21)$ and $a_{f\left(t_{i_{1}+1}\right)}=t_{i_{1}+1}$ by (6.19). Consequently, the equality

$$
t_{i_{0}-1}=t_{i_{1}+1}
$$

holds. Since $2 \leq i_{0}-1<i_{0}<i_{1}+1 \leq n$, equality (6.22) leads to a contradiction of the definition of cycles.

From (6.15) and (6.9) we obtain that

$$
w\left(\left\{t_{0}, t_{1}\right\}\right)=w\left(\left\{t_{1}, t_{2}\right\}\right)=\ldots=w\left(\left\{t_{n-1}, t_{n}\right\}\right)=\varepsilon_{1} .
$$

This chain of equalities evidently implies inequality (6.6). Hence, by Lemma 6.12, the weight $w$ defined by (6.9), is pseudometrizable. Using this lemma again, we obtain that $w$ is metrizable if the inequality

$$
\inf \left\{w(F): F \in P_{x, y}\right\}>0
$$

holds for all distinct $x, y \in X$. Let us prove (6.23). Let $x$ and $y$ be two distinct points of $X$ and let $F=\left(t_{0}, \ldots, t_{n}\right)$ be a path joining $x$ and $y$ in $G, x=t_{0}$ and 
$y=t_{n}$. If there exists $i \in I$ such that $\left\{t_{k}, t_{k+1}\right\} \subseteq X_{i}$ for every $k \in\{0, \ldots, n-1\}$, then $x, y \in X_{i}$ and using the triangle equality, we see that

$$
w(F)=\sum_{k=0}^{n-1} w\left(\left\{t_{k}, t_{k+1}\right\}\right)=\sum_{k=0}^{n-1} d_{X_{i}}\left(t_{k}, t_{k+1}\right) \geq d_{X_{i}}(x, y)>0 .
$$

(Note that $x$ and $y$ are distinct points of $X$.) Otherwise, there is $k_{0} \in\{0, \ldots, n-1\}$ such that $f\left(t_{k_{0}}\right) \neq f\left(t_{k_{0}}+1\right)$ and similarly to (6.13) we obtain

$$
t_{k_{0}}=a_{f\left(t_{k_{0}}\right)} \quad \text { and } \quad t_{k_{0}+1}=a_{f\left(t_{k_{0}+1}\right)} .
$$

Since $w$ is nonnegative, (6.24) and (6.9) imply that

$$
w(F)=\sum_{k=0}^{n-1} w\left(\left\{t_{k}, t_{k+1}\right\}\right) \geq w\left(\left\{t_{k_{0}}, t_{k_{0}+1}\right\}\right)=w\left(\left\{a_{f\left(t_{k_{0}}\right)}, a_{f\left(t_{k_{0}+1}\right)}\right\}\right)=\varepsilon_{1} .
$$

Thus, for every $F \in P_{x, y}$, we obtain

$$
w(F) \geq d_{X_{i}}(x, y)
$$

if there is $i \in I$ such that $x, y \in X_{i}$, and

$$
w(F) \geq \varepsilon_{1}
$$

if $x \in X_{i}$ and $y \in Y_{j}$ with distinct $i, j \in I$. Inequality (6.23) follows from (6.25) and (6.26). Hence the weight $w$ is metrizable.

Let $d_{w, G}$ be the weighted shortest path metric defined by (6.5). (Note that inequality (6.23) implies that $d_{w, G}$ really is a metric.) Using the last statement of Lemma 6.12 , we see that the metric space $X$ with the metric $d_{X}=d_{w, G}$ is $\mathfrak{M}$ universal. In accordance with Theorem 6.8 , to prove that $X$ is minimal $\mathfrak{M}$-universal it suffices to show that for every $i \in I$ there is a unique $X^{i} \subseteq X$ such that

$$
Y_{i} \simeq X^{i}
$$

It is clear that $Y_{i} \simeq X_{i}$ for every $i \in I$. Suppose there is $i_{0} \in I$ such that $X^{i_{0}} \neq X_{i_{0}}$ and $Y_{i_{0}} \simeq X^{i_{0}}$. If $X^{i_{0}} \subseteq X_{i_{0}}$, then we have $X^{i_{0}}=X_{i_{0}}$ because $X^{i_{0}} \subseteq X_{i_{0}} \simeq Y_{i_{0}}$ and $Y_{i_{0}}$ is unshifted. Hence there is a point $x_{1} \in X^{i_{0}}$ and an index $i_{1} \in I$ such that $x_{1} \in X_{i_{1}}$ and $i_{1} \neq i_{0}$. If the inclusion $X^{i_{0}} \subseteq X_{i_{1}}$ holds, then $Y_{i_{0}} \hookrightarrow X_{i_{1}}$ and consequently $Y_{i_{0}} \hookrightarrow Y_{i_{1}}$. This leads to a contradiction with the condition that $Y_{i_{0}}$ and $Y_{i_{1}}$ are incomparable if $i_{0} \neq i_{1}$. Consequently, there is $i_{2} \in I$ and $x_{2} \in X^{i_{0}}$ such that $x_{2} \in X_{i_{2}}$ with $i_{2} \neq i_{1}$. Since $x_{1}, x_{2} \in X^{i_{0}}, X^{i_{0}} \simeq Y_{i_{0}}$ and $Y_{i_{0}}$ is $\varepsilon$-connected, there is a finite sequence $t_{0}, \ldots, t_{n}$ of points from $X^{i_{0}}$ with

$$
t_{i} \in X, t_{0}=x_{1}, t_{n}=x_{2}, \quad \text { and } \quad d_{X}\left(t_{i}, t_{i+1}\right)<\varepsilon
$$

for every $i \in\{0, \ldots, n-1\}$. Since $t_{0}=x_{1}$ and $t_{n}=x_{2}$, we have $t_{0} \in X_{i_{1}}$ and $t_{n} \in X_{i_{2}}$ with $i_{1} \neq i_{2}$. Consequently, we can find $i \in\{0, \ldots, n-1\}$ such that $f\left(t_{i}\right) \neq f\left(t_{i+1}\right)$. Now using (6.26), we obtain

$$
d_{X}\left(t_{i}, t_{i+1}\right)=d_{w, G}\left(t_{i}, t_{i+1}\right)=\inf \left\{w(F): F \in P_{t_{i}, t_{i+1}}\right\} \geq \varepsilon_{1},
$$

contrary to (6.27). Hence if $X^{i_{0}} \neq X_{i_{0}}$, then $X^{i_{0}} \neq Y_{i_{0}}$ holds. It follows that $X$ is minimal $\mathfrak{M}$-universal.

It is well known that every connected metric space is $\varepsilon$-connected for all $\varepsilon>0$. Hence, Theorem 6.10 entails the following corollary. 
Corollary 6.13. Let $\mathfrak{M}$ be a non-empty set of connected, non-empty metric spaces $Y$. Suppose that all $Y$ are unshifted and pairwise incomparable, then there is a metric $d_{X}$ on

$$
X=\coprod_{Y \in \mathfrak{M}} Y
$$

such that $\left(X, d_{X}\right)$ is minimal $\mathfrak{M}$-universal.

The following corollary can be considered as a special case of Corollary 6.13.

Corollary 6.14. Let $n$ be a positive integer number and let $\mathcal{N}_{n}$ be the class of all normed $n$-dimensional linear spaces over the field $\mathbf{R}$. Then there is a set $\mathfrak{M} \subseteq \mathcal{N}_{n}$ and a disjoint union

$$
X=\coprod_{Y \in \mathfrak{M}} Y
$$

such that $\mathfrak{M}$ is a minimal $\mathcal{N}_{n}$-universal set of metric spaces and $X$ is a minimal $\mathcal{N}_{n}$-universal metric space.

Proof. The existence of minimal $\mathcal{N}_{n}$-universal $\mathfrak{M} \subseteq \mathcal{N}_{n}$ was proved in Proposition 5.26. Since every normed vector space over $\mathbf{R}$ is connected, the existence of a minimal $\mathcal{N}_{n}$-universal disjoint union $\coprod_{Y \in \mathfrak{M}} Y$ follows from Corollary 6.13.

\section{Metric betweenness and minimal universality}

The ternary "betweenness" relation was introduced in explicit form by Hilbert in [18]. In the theory of metric spaces, the notion of "metric betweenness", which is essential in the present section, first appeared at Menger's paper [33] in the following form.

Definition 7.1. Let $X$ be a metric space and let $x, y$ and $z$ be points of $X$. One says that $y$ lies between $x$ and $z$ if

$$
d_{X}(x, z)=d_{X}(x, y)+d_{X}(y, z) .
$$

The "betweenness" relation thus defined is fundamental for the theory of geodesics on metric spaces (see, e.g., [35]), and it naturally arises in the studies of the best approximations in metric spaces [14].

Characteristic properties of the ternary relations that are "metric betweenness" relations for (real-valued) metrics were determined by Wald in [52]. Later, the problem of "metrization" of betweenness relations (not necessarily by real-valued metrics) was considered in [32], [34] and [39]. An infinitesimal version of the metric betweenness was obtained in [4] and [12].

Let $X$ be a metric space. It is easy to verify that, for every three points $x, y, z \in$ $X$, the equality

$$
2 \max \left\{d_{X}(x, y), d_{X}(x, z), d_{X}(y, z)\right\}=d_{X}(x, y)+d_{X}(x, z)+d_{X}(y, z)
$$

holds if and only if one of these points lies between the other two points. The necessary and sufficient condition for (7.1) is the equality of the Cayley-Menger determinant to zero:

$$
\operatorname{det}\left|\begin{array}{cccc}
0 & d_{X}^{2}(x, y) & d_{X}^{2}(x, z) & 1 \\
d_{X}^{2}(y, x) & 0 & d_{X}^{2}(y, z) & 1 \\
d_{X}^{2}(z, x) & d_{X}^{2}(z, y) & 0 & 1 \\
1 & 1 & 1 & 0
\end{array}\right|=0
$$


(see, e.g., [3, p. 290]).

Let $\mathfrak{M} \mathfrak{B}$ be the class of metric spaces $X$, with points $x, y, z$ satisfying (7.1). Thus $X \in \mathfrak{M B}$ if and only if, among any three points of $X$, there exists a point that lies between the others.

Let $\mathbf{R}$ denote the real line with the standard metric $d_{\mathbf{R}}(x, y)=|x-y|$. It is clear that $\mathbf{R} \in \mathfrak{M B}$.

Definition 7.2. A metric space $X$ is called a pseudo-linear quadruple if $X \nrightarrow \mathbf{R}$ and $A \hookrightarrow \mathbf{R}$ for every $A \subseteq X$ with $|A| \leqslant 3$.

Lemma 7.3. A metric space $X$ is a pseudo-linear quadruple if and only if $|X|=4$ and the points of $X$ may be labeled $p_{0}, p_{1}, p_{2}, p_{3}$ so that

$$
d_{X}\left(p_{0}, p_{1}\right)=d_{X}\left(p_{2}, p_{3}\right), \quad d_{X}\left(p_{1}, p_{2}\right)=d_{X}\left(p_{3}, p_{0}\right)
$$

and

$$
d_{X}\left(p_{0}, p_{2}\right)=d_{X}\left(p_{0}, p_{1}\right)+d_{X}\left(p_{1}, p_{2}\right)=d_{X}\left(p_{1}, p_{3}\right) .
$$

Definition 7.2 and Lemma 7.3 are the one-dimensional cases of much more general Definition 44.1 and Theorem 45.4 of Blumenthal's book [5] dealing with isometric embeddings of semimetric spaces in the Euclidean spaces $E^{n}$ with an arbitrary finite $n$. An elementary proof of Lemma 7.3 can be found in [12].

Write

$$
\mathfrak{M B}^{5}:=\{X \in \mathfrak{M B}:|X| \geq 5\} .
$$

Proposition 7.4. The space $\mathbf{R}$ is minimal $\mathfrak{M P}^{5}$-universal. If $X$ is universal for $\mathfrak{M B}^{5}$ and $X \in \mathfrak{M B}^{5}$ or if $X$ is minimal $\mathfrak{M B}^{5}$-universal, then $X \simeq \mathbf{R}$.

Proof. The universality of $\mathbf{R}$ for $\mathfrak{M B}^{5}$ follows from Lemma 7.3. Since $\mathbf{R}$ is boundedly compact, homogeneous and belongs to $\mathfrak{M B}^{5}$, Theorem 3.26 implies that $\mathbf{R}$ is minimal $\mathfrak{M B}^{5}$-universal and $\mathbf{R} \simeq X$ for every $\mathfrak{M B}^{5}$-universal $X \in \mathfrak{M B}^{5}$ and every minimal $\mathfrak{M B}^{5}$-universal $X$.

Let $\mathfrak{F}_{2}$ be the class of all metric spaces $X$ with $|X| \leqslant 2$. The class $\mathfrak{F}_{2}$ admits a minimal universal set $\mathfrak{F}_{2}^{1} \subseteq \mathfrak{F}_{2}$. We can construct such set by the rule: a metric space $Y$ belongs to $\mathfrak{F}_{2}^{1}$ if and only if there is $t \in(0, \infty)$ such that $Y=\{0, t\}$. Thus, by definition, we have

$$
\mathfrak{F}_{2}^{1}=\left\{Y_{t}: t \in(0, \infty)\right\}
$$

where $Y_{t}=\{0, t\}$. It is evident that every $Y_{t} \in \mathfrak{F}_{2}^{1}$ is unshifted and $Y_{t_{1}} \nrightarrow Y_{t_{2}}$ if $t_{1} \neq t_{2}$.

Proposition 7.5. Let $T$ be a non-empty subset of $(0, \infty)$ and let

$$
\mathfrak{A}=\left\{Y_{t}: t \in T\right\} .
$$

The following conditions are equivalent.

(i) For every $Z \in \mathfrak{A}$ there is $Y \in \mathfrak{F}_{2}^{1} \backslash \mathfrak{A}$ such that

$$
\operatorname{diam} Z<\operatorname{diam} Y \text {. }
$$

(ii) There is a disjoint union

$$
X=\coprod_{t \in T} Y_{t}
$$

with a metric $d_{X}$ such that $\left(X, d_{X}\right)$ is minimal $\mathfrak{A}$-universal. 
Proof. Let (i) hold. It is easy to see that (i) holds if and only if the set $(0, \infty) \backslash T$ is unbounded. If $T$ is bounded, then all $Y_{t}, t \in T$ are $\varepsilon$-connected metric spaces with

$$
\varepsilon=\sup _{t \in T}\left(\operatorname{diam} Y_{t}\right) \text {. }
$$

In this case condition (ii) follows from Theorem 6.10. If $T$ and $(0, \infty) \backslash T$ are both unbounded, then there is a sequence $\left(p_{n}\right)_{n \in \mathbf{N}}$ such that

$$
p_{1}=0, p_{n} \in(0, \infty) \backslash T, \quad p_{n-1}<p_{n}, \quad \text { and } \quad\left(p_{n-1}, p_{n}\right) \cap T \neq \varnothing
$$

for every $n \geqslant 2$ and

$$
\bigcup_{n=2}^{\infty} T \cap\left(p_{n-1}, p_{n}\right)=T \text {. }
$$

Let $\left\{X_{t}: t \in T\right\}$ be a disjoint family of metric spaces with $X_{t} \simeq Y_{t}$ for every $t \in T$. Write

$$
X=\bigcup_{t \in T} X_{t}
$$

Let us define a function $d_{X}: X \times X \rightarrow \mathbf{R}^{+}$as

$$
d_{X}(x, y)= \begin{cases}d_{X_{t}}(x, y) & \text { if there is } t \in T \text { such that } x, y \in X_{t} \\ p_{n} & \text { if } x \in X_{t_{1}}, y \in X_{t_{2}}, t_{1} \neq t_{2} \\ & \text { and } \max \left\{t_{1}, t_{2}\right\} \in\left(p_{n-1}, p_{n}\right) .\end{cases}
$$

The function $d_{X}$ is symmetric and non-negative and, moreover,

$$
\left(d_{X}(x, y)=0\right) \Longleftrightarrow(x=y)
$$

holds for all $x, y \in X$. We claim also that the strong triangle inequality

$$
d_{X}(x, y) \leqslant \max \left\{d_{X}(x, z), d_{X}(z, y)\right\}
$$

is valid for all $x, y, z \in X$. Indeed, if there is $t \in T$ such that $x, y, z \in X_{t}$, then there is nothing to prove. In the case when $x \in X_{t_{1}}, y \in X_{t_{2}}, z \in X_{t_{3}}$ and

$$
\min \left\{t_{1}, t_{2}, t_{3}\right\} \neq \max \left\{t_{1}, t_{2}, t_{3}\right\} \quad \text { and } \max \left\{t_{1}, t_{2}, t_{3}\right\} \in\left(p_{n-1}, p_{n}\right)
$$

we have

$$
d_{X}(x, y) \leqslant p_{n} \quad \text { and } \max \left\{d_{X}(x, z), d_{X}(z, y)\right\}=p_{n} .
$$

Thus, (7.2) holds for all $x, y, z \in X$. Consequently $d_{X}$ is an ultrametric.

Since $p_{n} \in(0, \infty) \backslash T$ for every $n \geq 2$, it follows from (7.2) that, for every $t \in T$, there is a unique two-point set $\{x, y\} \subseteq X$ such that $d_{X}(x, y)=t$. In the correspondence with Theorem 6.8 the disjoint union $X=\coprod_{t \in T} Y_{t}$ with the metric $d_{X}$ is a minimal $\mathfrak{A}$-universal metric space. The implication (i) $\Longrightarrow$ (ii) follows.

Suppose now that (ii) holds but (i) does not hold. As was noted above, (i) holds if and only if the set $(0, \infty) \backslash T$ is unbounded. Consequently there is $t_{0} \in(0, \infty)$ such that

$$
(0, \infty) \backslash T \subseteq\left(0, t_{0}\right)
$$

Let $t_{1} \in\left(2 t_{0}, \infty\right)$. Inclusion (7.3) implies that $t_{1} \in T$. Hence $Y_{t_{1}} \in \mathfrak{A}$ holds. In the correspondence with the supposition, a disjoint union $X=\coprod_{t \in T} Y_{t}$ with a metric $d_{X}$ is a minimal $\mathfrak{A}$-universal metric space. Using (7.3) and Theorem 6.8 we see that for every $t^{\prime}>t_{0}$ there is a unique two-point set $\left\{x^{\prime}, y^{\prime}\right\} \subseteq X$ such that $d_{X}\left(x^{\prime}, y^{\prime}\right)=t^{\prime}$ and, moreover, if $t^{\prime \prime}>t_{0}$ and

$$
\left\{x^{\prime \prime}, y^{\prime \prime}\right\} \subseteq X, \quad d_{X}\left(x^{\prime \prime}, y^{\prime \prime}\right)=t^{\prime \prime},
$$


then

$$
\left\{x^{\prime} y^{\prime}\right\} \cap\left\{x^{\prime \prime}, y^{\prime \prime}\right\}=\varnothing .
$$

Note that (7.4) follows from the above mentioned uniqueness and Definition 6.1. Let $\left\{x_{1}, y_{1}\right\} \subseteq X$ and $d_{X}\left(x_{1}, y_{1}\right)=t_{1}$. Let $z_{1}$ be an arbitrary point of $X \backslash\left\{x_{1}, y_{1}\right\}$. From the triangle inequality it follows that we have at least one from the inequalities

$$
d_{X}\left(x_{1}, z_{1}\right) \geqslant \frac{1}{2} t_{1} \quad \text { or } \quad d_{X}\left(y_{1}, z_{1}\right) \geqslant \frac{1}{2} t_{1}
$$

Without loss of generality, we can suppose that the first inequality of (7.5) holds. Then we have

and

$$
\left\{x_{1}, y_{1}\right\} \subseteq X, \quad\left\{x_{1}, z_{1}\right\} \subseteq X
$$

$$
d_{X}\left(x_{1}, y_{1}\right) \in T, \quad d_{X}\left(x_{1}, z_{1}\right) \in T
$$

and

$$
\left\{x_{1}, y_{1}\right\} \cap\left\{x_{1}, z_{1}\right\}=\left\{x_{1}\right\}
$$

contrary to (7.4). The implication (ii) $\Longrightarrow$ (i) follows.

Corollary 7.6. The set $\mathfrak{F}_{2}^{1}$ has the following properties.

(i) Every $Y \in \mathfrak{F}_{2}^{1}$ is an unshifted metric space and if $Y_{t_{1}}$ and $Y_{t_{2}}$ are distinct, then $Y_{t_{1}} \leftrightarrow Y_{t_{2}}$

(ii) Any disjoint union $\coprod_{Y \in \mathfrak{F}_{2}^{1}} Y$ is universal but not minimal universal for $\mathfrak{F}_{2}^{1}$.

(iii) There are subsets $\mathfrak{A}_{1}$ and $\mathfrak{A}_{2}$ of $\mathfrak{F}_{2}^{1}$ such that

$$
\mathfrak{A}_{1} \cap \mathfrak{A}_{2}=\varnothing, \quad \mathfrak{A}_{1} \cup \mathfrak{A}_{2}=\mathfrak{F}_{2}^{1}
$$

and the disjoint unions

$$
\coprod_{Y \in \mathfrak{A}_{1}} Y \text { and } \coprod_{Y \in \mathfrak{A}_{2}} Y
$$

are minimal universal for $\mathfrak{A}_{1}$ and $\mathfrak{A}_{2}$ respectively.

Thus, there is no minimal $\mathfrak{F}_{2}$-universal metric spaces $X$ of the form

$$
X=\coprod_{Y \in \mathfrak{F}_{2}^{1}} Y .
$$

Nevertheless, the next simple example of a minimal $\mathfrak{F}_{2}$-universal metric space was constructed by Wozniak in 2008.

Example 7.7. [20] The set

$$
X=(-1,0) \cup\left(\bigcup_{n=1}^{\infty}\{n\}\right)
$$

with the metric induced from $\mathbf{R}$ is a minimal $\mathfrak{F}_{2}$-universal metric space (see Figure 2 ). The minimality of $X$ is, in fact, a consequence of the uniqueness of the representation of $x \in(0, \infty)$ in the form

$$
x=n+t
$$

where $n \in \mathbf{N} \cup\{0\}$ and $t \in[0,1)$. For the proof note that if (7.6) holds, then

$$
n=\lfloor x\rfloor \text { and } t=x-\lceil x\rceil \text {. }
$$

Remark 7.8. An example of a non-separable minimal $\mathfrak{F}_{2}$-universal metric space was constructed by Holstýnski in [20]. 


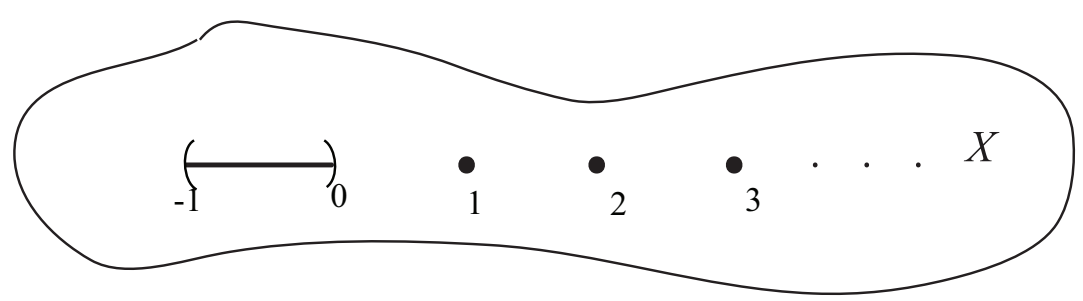

Figure 2. The $\mathfrak{F}_{2}$-universal space $\mathbf{R}$ contains the minimal $\mathfrak{F}_{2}$-universal subspace $X$.

The next our goal is the building of a minimal $\mathfrak{M B}$-universal metric space. Let $\mathfrak{P} \mathfrak{L}$ be the class of all pseudo-linear quadruples. It is clear that $\mathfrak{P} \mathfrak{L} \subseteq \mathfrak{M B}$ and we begin by constructing of a minimal $\mathfrak{P} \mathfrak{L}$-universal metric space.

Let $X$ be a metric space and let $A \subseteq X$. We shall say that a two-element subset $\{a, b\}$ of $A$ is a diametrical pair for $A$ if

$$
d_{X}(a, b)=\operatorname{diam} A
$$

where as usual

$$
\operatorname{diam} A=\sup \left\{d_{X}(x, y): x, y \in A\right\} .
$$

A point $a \in X$ is a diametrical point for $A$ if $a \in A$ and there is $b \in A$ such that $\{a, b\}$ is a diametrical pair for $A$.

Lemma 7.9. If $X$ is a pseudo-linear quadruple, then every $x \in X$ is a diametrical point for $X$ and the number of diametrical pairs for $X$ is two. Furthermore, if $Y$ is also a pseudo-linear quadruple and there are $\left\{x_{1}, x_{2}, x_{3}\right\} \subseteq X$ and $\left\{y_{1}, y_{2}, y_{3}\right\} \subseteq Y$ such that $\left\{x_{1}, x_{2}, x_{3}\right\} \simeq\left\{y_{1}, y_{2}, y_{3}\right\}$, then $X \simeq Y$.

A proof is immediate from Lemma 7.3.

As in the case of the class $\mathfrak{F}_{2}$, there is a minimal $\mathfrak{P} \mathfrak{L}$-universal disjoint set $\mathfrak{P} \mathfrak{L}^{1} \subseteq$ $\mathfrak{P} \mathfrak{L}$. Let

$$
X:=\bigcup_{Y \in \mathfrak{P L}^{1}} Y
$$

and let $d_{X}: X \times X \rightarrow \mathbf{R}^{+}$be a function such that

$$
d_{X}(z, y)= \begin{cases}d_{Y}(z, y) & \text { if there is } Y \in \mathfrak{P L}^{1} \text { with } z, y \in Y, \\ \max \{\operatorname{diam} Y, \operatorname{diam} Z\} & \text { if there are distinct } Y, Z \in \mathfrak{P} \mathfrak{L}^{1} \\ & \text { with } y \in Y, z \in Z\end{cases}
$$

Note that (7.8) is correct because $\mathfrak{P} \mathfrak{L}^{1}$ is disjoint.

Lemma 7.10. If $X$ and $d_{X}: X \times X \rightarrow \mathbf{R}^{+}$are defined as in (7.7) and (7.8) respectively, then $d_{X}$ is a metric on $X$.

Proof. It suffices to show that the strong triangle inequality

$$
d_{X}(y, z) \leq \max \left\{d_{X}(y, w), d_{X}(w, z)\right\}
$$

holds if $y \in Y, z \in Z, w \in W$ and $Y, Z, W \in \mathfrak{P L}^{1}$ and at least one from the conditions

$$
Y \neq Z, \quad Y \neq W \quad \text { and } \quad Z \neq W
$$

is valid. There exist the following four possibilities:

(i) $Y \neq Z$ and $Y \neq W$ and $Z \neq W$;

(ii) $Y \neq Z$ and $Z=W$;

(iii) $Y \neq Z$ and $Y=W$; 
(iv) $Y=Z$ and $Z \neq W$.

In the case when (i) holds, inequality (7.9) is evident. Suppose we have (ii). Then, the equalities

$$
\max \{\operatorname{diam} Y, \operatorname{diam} Z\}=d_{X}(y, z)
$$

and

$$
\max \{\operatorname{diam} Y, \operatorname{diam} Z\}=\max \{\operatorname{diam} W, \operatorname{diam} Z\}=d_{X}(y, w)
$$

hold which implies (7.9). Case (iii) is similar to (ii). Let (iv) be valid. Then using (7.8), we obtain

$$
d_{X}(y, z) \leq d_{Z}(y, z) \leq \operatorname{diam} Z .
$$

Moreover, from $Z \neq W$ and (7.8) it follows that

$$
d_{X}(z, w)=\max \{\operatorname{diam} Z, \operatorname{diam} W\} .
$$

Inequality (7.9) follows.

Remark 7.11. The metric $d_{X}$ in the above lemma is not an ultrametric.

Proposition 7.12. If $X$ and $d_{X}: X \times X \rightarrow \mathbf{R}^{+}$are defined as in (7.7) and (7.8) respectively, then $\left(X, d_{X}\right)$ is a minimal $\mathfrak{P} \mathfrak{L}$-universal metric space.

Proof. It follows directly from (7.8) and Lemma 7.10 , that $\left(X, d_{X}\right)$ is a disjoint union of $Y \in \mathfrak{P} \mathfrak{L}^{1}$. By Theorem $6.8,\left(X, d_{X}\right)$ is minimal $\mathfrak{P} \mathfrak{L}^{1}$-universal if and only if, for every $Y \in \mathfrak{P}^{\mathfrak{1}}$, there is a unique $X_{Y} \subseteq X$ such that $X_{Y} \simeq Y$. The last condition holds if for all distinct $y_{i} \in Y_{i}, i=1, \ldots, 4$ with $\left\{y_{1}, y_{2}, y_{3}, y_{4}\right\} \in \mathfrak{P} \mathfrak{L}$ we have

$$
Y_{1}=Y_{2}=Y_{3}=Y_{4} \text {. }
$$

Suppose $y_{i} \in Y_{i}, i=1, \ldots, 4$ but (7.10) does not hold. Then, as was shown in the proof of Lemma 7.10 there are three distinct points $x_{1}, x_{2}, x_{3} \in\left\{y_{1}, y_{2}, y_{3}, y_{4}\right\}$, which form an isosceles triangle with the base that is not longer than its legs. Hence the number of diametrical pairs of $\left\{x_{1}, x_{2}, x_{3}\right\}$ is more than or equal to two. Consequently, $\left\{x_{1}, x_{2}, x_{3}\right\} \notin \mathfrak{M B}$. It follows from Definition 7.2 that $\left\{y_{1}, y_{2}, y_{3}, y_{4}\right\} \notin \mathfrak{P} \mathfrak{L}$. Thus, $\left(X, d_{X}\right)$ is minimal $\mathfrak{P} \mathfrak{L}^{1}$-universal and, consequently, minimal $\mathfrak{P} \mathfrak{L}$-universal.

Now we are ready to construct a minimal $\mathfrak{M B}$-universal metric space. Let $p \in \mathbf{R}$, $r \in(0, \infty)$ and $b \in X$, where

$$
X=\bigcup_{Y \in \mathfrak{P L}^{1}} Y .
$$

For simplicity, we may suppose $X \cap \mathbf{R}=\varnothing$. Write $M:=X \cup \mathbf{R}$ and define a symmetric function $d_{M}: M \times M \rightarrow \mathbf{R}^{+}$such that

$$
d_{M}(x, y)= \begin{cases}d_{X}(x, y) & \text { if } x, y \in X, \\ |x-y| & \text { if } x, y \in \mathbf{R}, \\ |x-p|+r+d_{X}(b, y) & \text { if } x \in \mathbf{R} \text { and } y \in X .\end{cases}
$$

Lemma 7.13. The function $d_{M}: M \times M \rightarrow \mathbf{R}^{+}$is a metric on $M$.

A proof of Lemma 7.13 can be obtained directly from (7.11). Note only, that $d_{M}$ is the weighted shortest-path metric for the weighted graph $(G, w)$ with the vertex set $V(G)=M$, and the edge set

$$
E(G)=\{\{x, y\}: x, y \in X, x \neq y\} \cup\{\{p, b\}\} \cup\{\{x, y\}: x, y \in \mathbf{R}, x \neq y\},
$$


and the weight

$$
w(\{x, y\})= \begin{cases}d_{X}(x, y) & \text { if }\{x, y\} \subseteq X, \\ |x-y| & \text { if }\{x, y\} \subseteq \mathbf{R}, \\ r & \text { if }\{x, y\}=\{p, b\} .\end{cases}
$$

Lemma 7.14. Let $x, y \in \mathbf{R}, x<y$ and let $z \in X$. If $\{x, y, z\} \in \mathfrak{M B}$, then $p$ does not belong to the interval $(x, y)=\{t \in \mathbf{R}: x<t<y\}$.

Proof. Suppose $\{x, y, z\} \in \mathfrak{M} \mathfrak{B}$. Then there exist the following three possibilities:

(i) $z$ lies between $x$ and $y$,

$$
d_{M}(x, y)=d_{M}(x, z)+d_{M}(z, y)
$$

(ii) $y$ lies between $x$ and $z$,

$$
d_{M}(x, z)=d_{M}(x, y)+d_{M}(y, z)
$$

(iii) $x$ lies between $y$ and $z$,

$$
d_{M}(y, z)=d_{M}(y, x)+d_{M}(x, z) .
$$

For $p \in(x, y)$, equality $(7.12)$ can be written in the form

$$
|x-y|=\left(|x-p|+r+d_{X}(b, z)\right)+\left(|y-p|+r+d_{X}(b, z)\right)
$$

(see Figure 3 below). The condition $p \in(x, y)$ implies

$$
|x-p|+|y-p|=|x-y| \text {. }
$$

Hence from (7.15) we obtain

$$
0=2\left(r+d_{X}(b, z)\right) \geq 2 r,
$$

contrary to the inequality $r>0$. If we have (7.13), then

$$
|x-p|+r+d_{X}(b, z)=|x-y|+|y-p|+z+d_{X}(b, z),
$$

so that

$$
|x-p|=|x-y|+|y-p| \text {. }
$$

The last equality and (7.16) imply

$$
|x-p|=|x-p|+2|y-p| .
$$

Hence $|y-p|=0$ holds. It is a contradiction with $p \in(x, y)$.

Case (iii) can be considered analogously to case (ii). Thus, $p \in(x, y)$ contradicts the supposition $\{x, y, z\} \in \mathfrak{M B}$.

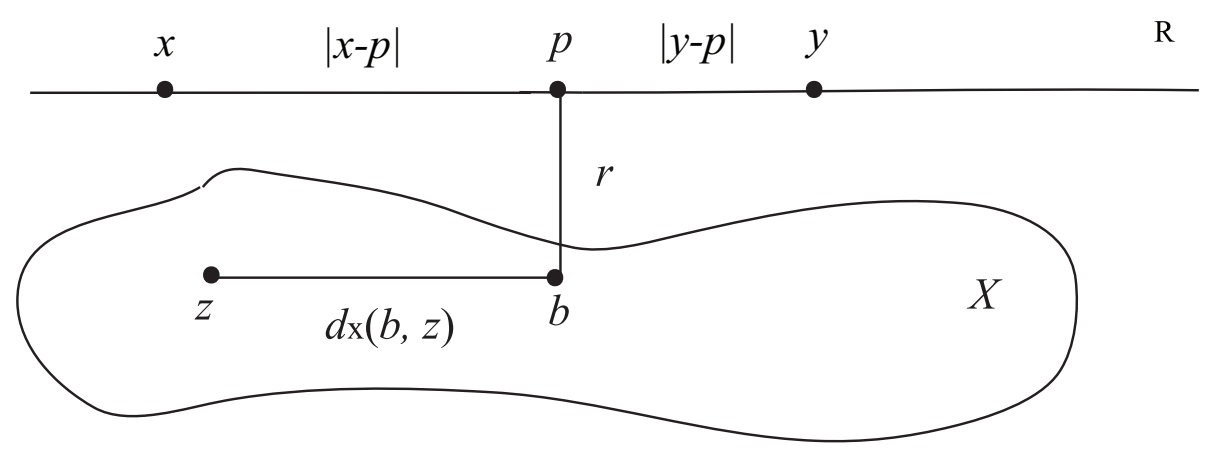

Figure 3. 
Theorem 7.15. The metric space $\left(M, d_{M}\right)$ is minimal $\mathfrak{M} \mathfrak{B}$-universal.

Proof. From Lemma 7.13 and the definitions of $d_{M}$ and $X$, it follows that

$$
M=X \sqcup \mathbf{R}=\left(\coprod_{Y \in \mathfrak{P} \mathfrak{L}^{1}} Y\right) \sqcup \mathbf{R} .
$$

Hence, $\{\mathbf{R}\} \cup \mathfrak{P} \mathfrak{L} \hookrightarrow M$. Moreover, using Definition 7.2 and Lemma 7.3, we see that, for every $Z \in \mathfrak{M B}$, either $Z \hookrightarrow \mathbf{R}$ or there is $Q \in \mathfrak{P} \mathfrak{L}$ such that $Q \simeq Z$. Consequently, $\mathfrak{M B} \hookrightarrow M$ and the set $\mathfrak{P} \mathfrak{L}^{1} \cup\{\mathbf{R}\}$ is a minimal $\mathfrak{M} \mathfrak{B}$-universal subset of $\mathfrak{M B}$. Hence, it suffices to show that $M$ is a minimal universal metric space for $\mathfrak{P} \mathfrak{L}^{1} \cup\{\mathbf{R}\}$. Condition (iii) of Theorem 6.8 implies that $M$ is minimal universal for $\mathfrak{P} \mathfrak{L}^{1} \cup\{\mathbf{R}\}$ if and only if for every $Z \in \mathfrak{P}^{1} \cup\{\mathbf{R}\}$ there is a unique $S_{Z} \subseteq M$, such that $S_{Z} \simeq Z$. Indeed, all $Z \in \mathfrak{P L}^{1}$ are unshifted as finite metric spaces and $\mathbf{R}$ is unshifted by Lemma 3.23. Moreover, it follows from Definition 7.2, Lemma 7.3 and the definition of $\mathfrak{P} \mathfrak{L}^{1}$ that any distinct $Z, W \in \mathfrak{P L}^{1} \cup\{\mathbf{R}\}$ are incomparable. Thus, conditions (i) and (ii) of Theorem 6.8 hold and it remains to prove condition (iii).

Let $S \subseteq M$ and $S \simeq \mathbf{R}$. We must show that $S=\mathbf{R}$. Since $\mathbf{R}$ is unshifted, it suffices to prove the inclusion $S \subseteq \mathbf{R}$. Suppose contrary that $S \cap X \neq \varnothing$, where $X=\coprod_{Y \in \mathfrak{P L}^{1}} Y$. Note that every point $x_{0} \in X$ is an isolated point of $M$. Indeed, the inequality

$$
d_{M}\left(x_{0}, y\right) \geq r
$$

holds if $y \in \mathbf{R}$ (see (7.11)) or we have the inequality

$$
d_{M}\left(x_{0}, y\right) \geq \operatorname{diam} Z_{0},
$$

if $x_{0} \in Z_{0} \in \mathfrak{P L}^{1}$ and $y \in Z_{1} \in \mathfrak{P} \mathfrak{L}^{1}, Z_{1} \neq Z_{0}$ (see (7.8)). Moreover,

$$
d_{M}\left(x_{0}, y\right) \geq \min \left\{d_{M}(x, y): x, y \in Z_{0}, x \neq y\right\}>0,
$$

holds if $x_{0}, y \in Z_{0} \in \mathfrak{P L}^{1}, x_{0} \neq y$. (Note that the second inequality in (7.17) holds, because $Z_{0}$ is finite.) Hence, if $S \cap X \neq \varnothing$, then $S$ contains an isolated point, which is impossible in the case $S \simeq \mathbf{R}$.

Suppose now that $S \subseteq M$ and $S \simeq Z$, with $Z \in \mathfrak{P L}^{1}$. If $S \subseteq X$, then the equality

$$
S=Z
$$

was, in fact, proved in the proof of Proposition 7.12. The inclusion $S \subseteq \mathbf{R}$ does not take place since $S \simeq Z$ and $Z \leftrightarrow \mathbf{R}$. Consequently, condition (iii) of Theorem 6.8 does not hold if and only if

$$
S \cap X \neq \varnothing \quad \text { and } \quad S \cap \mathbf{R} \neq \varnothing .
$$

Since $|S|=4$ and $S \cap X \neq \varnothing$, we can consider the following possibilities

$$
|S \cap \mathbf{R}|=3, \quad|S \cap \mathbf{R}|=2 \text { or }|S \cap \mathbf{R}|=1 .
$$

Case $|S \cap \mathbf{R}|=3$. Let $S \cap \mathbf{R}=\{x, y, s\}, x<y, S \cap X=\{z\}$ and let

$$
d_{M}(x, y)=\operatorname{diam}(S \cap \mathbf{R}) .
$$

Since $S \simeq Z$ and $Z \in \mathfrak{P L}^{1}$, the statement $\{x, y, z\} \in \mathfrak{M B}$ holds. Consequently, by Lemma 7.14, we have $p \notin(x, y)$ (see Figure 3). For example, if $x<y \leq p$, we obtain

$$
\operatorname{diam}\{x, y, s\}=d_{M}(x, y)=|x-y|<|x-y|+d_{M}(y, p)+r+d_{X}(b, z) \leq \operatorname{diam} S .
$$


Thus,

$$
\operatorname{diam}\{x, y, s\}<\operatorname{diam} S .
$$

Now note that Lemma 7.3 implies the equality $\operatorname{diam}\{x, y, s\}=\operatorname{diam} S$, contrary to (7.19).

Case $|S \cap \mathbf{R}|=2$. Let $S \cap \mathbf{R}=\{x, y\}$ and $S \cap X=\{s, z\}$. As in the case $|S \cap \mathbf{R}|=3$, we can show that $\{x, y\}$ is not a diametrical pair for $S$. Moreover, using Figure ??, it is easy to see that $x$ or $y$ is not a diametrical point for $X$, contrary to Lemma 7.9.

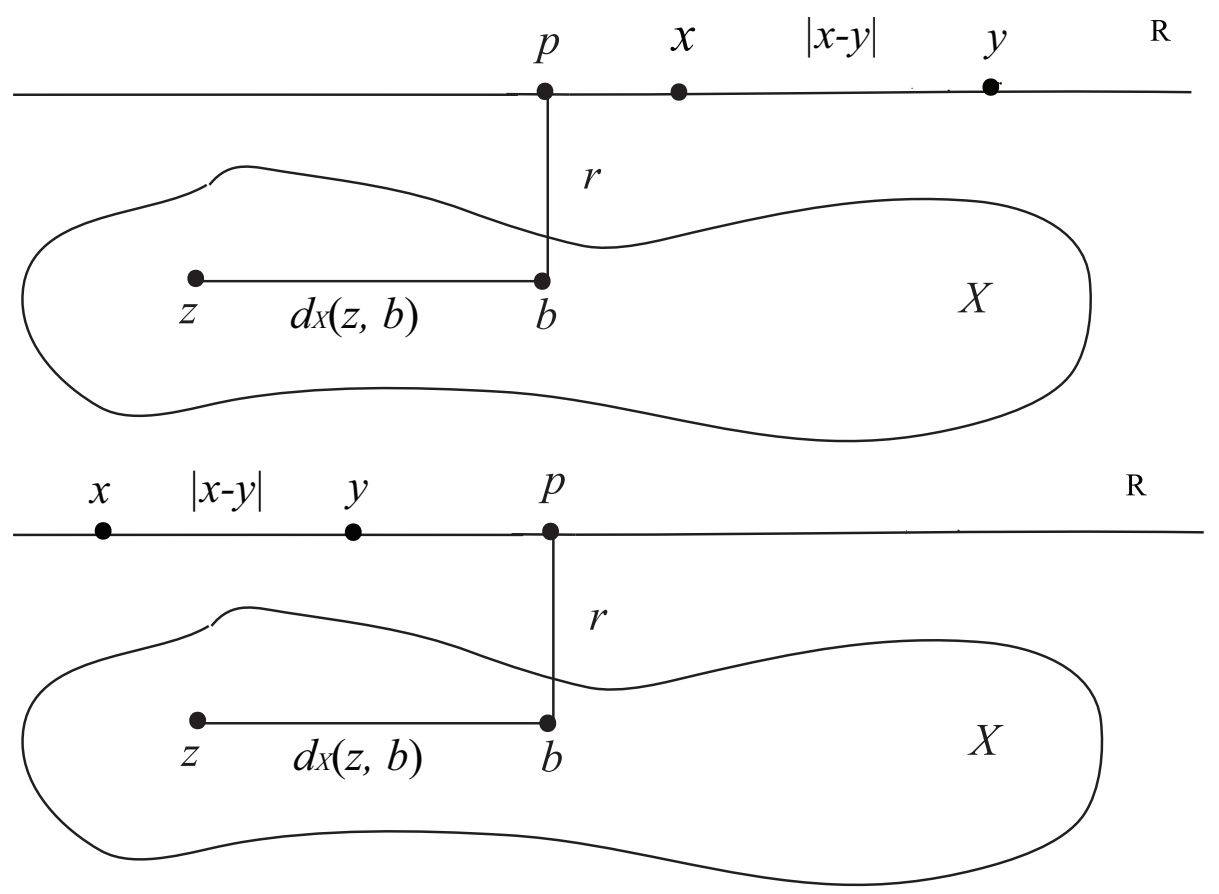

Figure 4 .

Case $|S \cap \mathbf{R}|=1$. Let $S \cap \mathbf{R}=\{x\}$ and $S \cap X=\{y, s, z\}$. Since $S \in \mathfrak{P} \mathfrak{L}$, we have

$$
\{y, s, z\} \in \mathfrak{M B} .
$$

As was shown in the proof of Proposition 7.12, statement (7.20) can be valid if and only if there is $W \in \mathfrak{P L}^{1}$ such that

$$
\{y, s, z\} \subseteq W
$$

(see (7.11)). By Lemma 7.9, we obtain that $W \simeq S$. Without loss of generality, we may suppose also that

$$
\operatorname{diam} S=d_{X}(y, z)
$$

If $b \notin W$, then using (7.8) we obtain that

$$
d_{M}(y, z)=d_{X}(y, z) \leq d_{X}(z, b)<d_{X}(z, b)+r+|p-x|=d_{M}(z, x) .
$$

Hence, $d_{M}(y, z)<d_{M}(z, x)$ holds, contrary to (7.11). Consequently, $b \in W$ holds. If $z=b$, then

$$
d_{M}(y, z)=d_{M}(y, b)<d_{X}(y, b)+r+|p-x|=d_{M}(y, x),
$$


that contradicts (7.21). Hence, we obtain $z \neq b$. Similarly we have $s \neq b$. Suppose $y=b$. Since $\{y, z\}$ is a diametrical pair for $S$, Lemma 7.9 implies that $\{x, s\}$ is also a diametrical pair for $S$. Using this lemma again, we obtain the inequality

$$
d_{M}(x, s)>d_{M}(x, y) .
$$

From the definition of $d_{M}$, it follows that

$$
d_{M}(x, s)=d_{M}(x, b)=|x-p|+r \text { and } d_{M}(x, y)=|x-p|+r+d_{M}(b, y),
$$

(see Figure 5). Thus, $d_{M}(x, s)<d_{M}(x, y)$, contrary to (7.22). Let us denote by $t$ the fourth point of the pseudo-linear quadruple $W \supseteq\{y, s, z\}$. Since $b \notin\{y, z, s\}$ and $b \in W$, we have $t=b$. Lemma 7.9 and $\{y, s, z\} \subseteq W \cap S$ imply that $W \simeq S$. In particular, the equalities

$$
d_{M}(t, s)=\operatorname{diam} W=\operatorname{diam} S=d_{M}(s, x)
$$

hold.

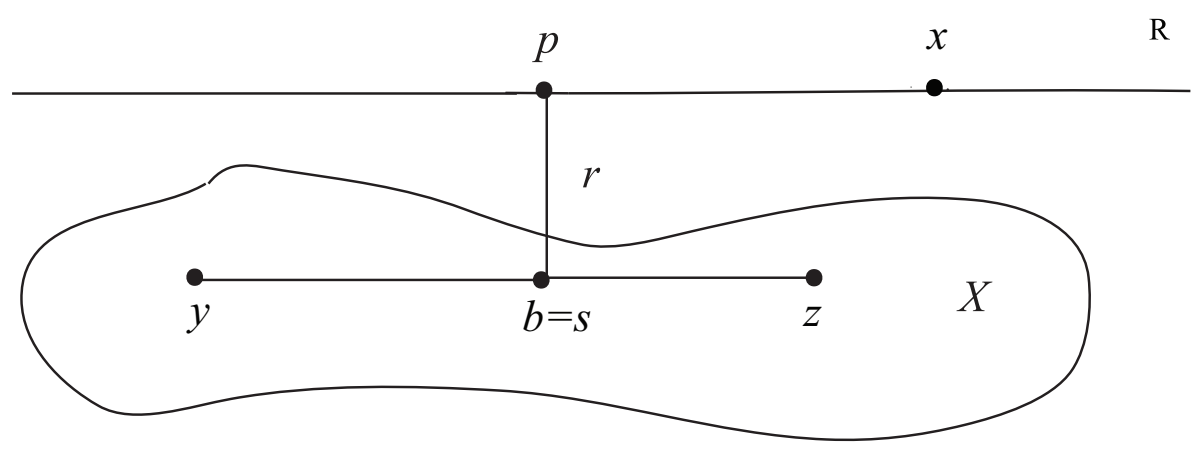

Figure 5 .

It follows that

$$
d_{M}(t, s)=d_{M}(s, x) .
$$

Now note that the last equality cannot hold, because

$$
d_{M}(x, s)=|x-p|+r+d_{M}(b, s)
$$

(see Figure 6).

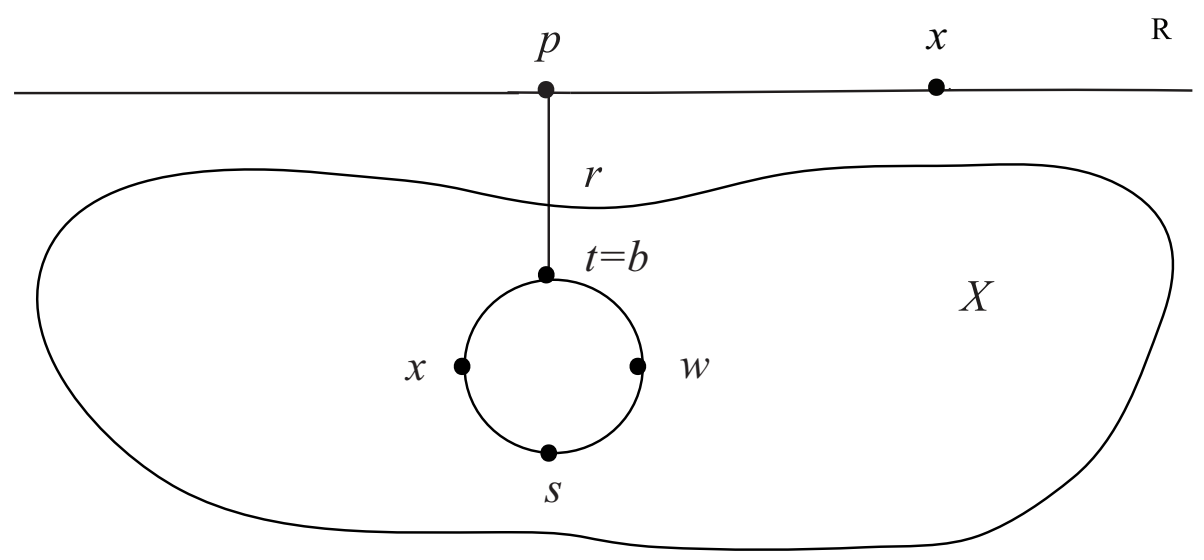

Figure 6 .

The following proposition gives us an example of a class $\mathfrak{M}$ of metric spaces for which all minimal $\mathfrak{M}$-universal metric spaces are isometric. 
Proposition 7.16. Let $\mathfrak{M}$ be the set of all closed intervals $[a, b] \subseteq \mathbf{R}$ with $|a-b|<1$ and let $I$ be the open interval $(0,1) \subseteq \mathbf{R}$. Then $I$ is a minimal $\mathfrak{M}$ universal metric space. Moreover, if $X$ is an arbitrary minimal $\mathfrak{M}$-universal metric space, then $X \simeq I$.

Proof. Let $X$ be a minimal $\mathfrak{M}$-universal metric space and let $p \in X$. Then for every $t \in(0,1)$ there is $f_{t}:[0, t] \hookrightarrow X$. Write $X_{t}=f_{t}([0, t])$. We claim that there exists $t_{0} \in(0,1)$ such that the statement

$$
p \in X_{t}
$$

holds for all $t \in\left[t_{0}, 1\right)$. Suppose the contrary and choose a sequence $\left(t_{n}\right)_{n \in \mathbf{N}}$ such that $t_{n} \in(0,1)$ and $\lim _{n \rightarrow \infty} t_{n}=1$ and $p \notin X_{t_{n}}$ for every $n \in \mathbf{N}$. Then

$$
Y=\bigcup_{n \in \mathbf{N}} X_{t_{n}}
$$

is a subspace of $X, p \notin Y$ and $\mathfrak{M} \hookrightarrow Y$. (The last follows from the equality $\lim _{n \rightarrow \infty} t_{n}=1$.) Hence $X$ is not minimal $\mathfrak{M}$-universal, contrary to the condition.

Now it is easy to show that $X \in \mathfrak{M B}$. Indeed, $X \in \mathfrak{M B}$ if and only if equality (7.1),

$$
2 \max \left\{d_{X}(x, y), d_{X}(y, z), d_{X}(z, x)\right\}=d_{X}(x, y)+d_{X}(y, z)+d_{X}(z, x)
$$

holds for all $x, y, z \in X$. Using the above proved claim we can find $X_{t_{*}}$ such that $x, y, z \in X_{t_{*}}$ for given $x, y, z \in X$. It is clear that $X_{t_{*}} \in \mathfrak{M B}$. Consequently equality (7.1) holds, so that $X \in \mathfrak{M B}$. Proposition 7.4 implies that $X$ is isometric to a subspace of $\mathbf{R}$. The metric space $X$ is connected because $X_{t}$ is connected for every $t \in(0,1)$ and, for given $x, y \in X$, there is $t_{*} \in(0,1)$ such that

$$
x, y \in X_{t_{*}} \subset X \text {. }
$$

A subset of $\mathbf{R}$ is connected if and only if this subset is an interval. Hence $X$ is an interval. Let us denote by $m(X)$ the length of $X$. It is clear that $\mathfrak{M} \leftrightarrow X$ if $m(X)<1$ and that $X$ is not minimal $\mathfrak{M}$-universal if $m(X)>1$. Consequently we have $m(X)=1$. Let $a$ and $b$ be the endpoints of $X$. Since $\mathfrak{M} \hookrightarrow X \backslash\{a, b\}$ and $X$ is minimal $\mathfrak{M}$-universal, the interval $X$ is open. Every open interval $(a, b)$ with $|a-b|=1$ is isometric to $I$. Thus $X \simeq I$ holds.

It still remains to note that $I$ is minimal $\mathfrak{M}$-universal. Indeed $\mathfrak{M} \hookrightarrow I$ is immediate. Now if $0<p<t<1$ and $1-p<t$, then we evidently have $[0, t] \in \mathfrak{M}$ and

$$
[0, t] \leadsto I \backslash\{p\} .
$$

Thus $I$ is a minimal $\mathfrak{M}$-universal metric space as required.

\section{Minimal universal subsets of $\mathbf{R}^{2}$ for the class of three-point metric spaces}

Let $\mathfrak{F}_{3}$ be the class of metric spaces $X$ with $|X| \leq 3$. It is a basic fact of the theory of metric spaces that the Euclidean plane $\mathbf{R}^{2}$ is a universal metric space for the class $\mathfrak{F}_{3}$. The main goal of the present section is to construct some minimal $\mathfrak{F}_{3}$-universal subsets of $\mathbf{R}^{2}$. Our first example of such subset is closely related to the so-called Fermat-Torricelli point of a triangle. Recall that $t \in \mathbf{R}^{2}$ is the Fermat-Torricelli point of a triangle $\{a, b, c\}$ if the inequality

$$
d_{\mathbf{R}^{2}}(a, t)+d_{\mathbf{R}^{2}}(b, t)+d_{\mathbf{R}^{2}}(c, t) \leq d_{\mathbf{R}^{2}}(a, x)+d_{\mathbf{R}^{2}}(b, x)+d_{\mathbf{R}^{2}}(c, x) .
$$


holds for every $x \in \mathbf{R}^{2}$. The geometric construction of the Fermat-Torricelli points can be found, for example, in Coxeter's book [8, p. 21-22].

If all angles of a given triangle $\{a, b, c\}$ are smaller than $\frac{2 \pi}{3}$, then the FermatTorricelli point $t$ satisfies the condition

$$
\angle a t b=\angle b t c=\angle c t a=\frac{2 \pi}{3} .
$$

For the convenience of the reader we repeat the following lemma here.

Lemma 8.1. Let $\{a, b, c\}$ be a triangle in $\mathbf{R}^{2}$. The following statements hold.

(i) All angles of $\{a, b, c\}$ are smaller than $\frac{2 \pi}{3}$ if and only if there is $t \in \mathbf{R}^{2}$ satisfying (8.2).

(ii) If $\mathbf{R}^{2}$ contains $t$ satisfying (8.2), then such $t$ is unique and belongs to the interior of the triangle $\{a, b, c\}$.

Proof. It suffices to note that for $x \in \mathbf{R}^{2}$ the equality $\angle a x b=\frac{2 \pi}{3}$ holds if and only if $x$ belongs to the symmetric lens $a f b f^{\prime} a$ (without points $a$ and $b$ ) where the lens is the union of the arcs $a f b$ and $a f^{\prime} b$ of two symmetric circles passing through $a$ and $b$ such that the angles made by the chord $a b$ and the tangents $a l$ and $a l^{\prime}$ are equal to $\frac{\pi}{3}$ (see Figure 7 ).

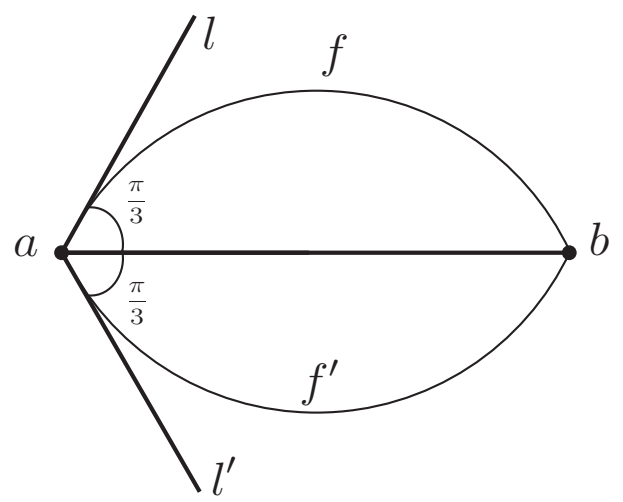

Figure 7. The locus of points $x$ with $\angle a x b=\frac{2 \pi}{3}$.

Consequently, if $\angle a b c \geqslant \frac{2 \pi}{3}$, then the lenses corresponding to the sides $[a, b]$ and $[b, c]$ meet at the point $b$ only. Otherwise there is a unique point $t$ satisfying (8.2) and lying in the interior of the triangle $\{a, b, c\}$.

Theorem 8.2. Let $X \subseteq \mathbf{R}^{2}$ be the union of some rays $\overrightarrow{o a}, \overrightarrow{o b}$, and $\overrightarrow{o c}$ with

$$
\angle a o b=\angle b o c=\angle c o a=\frac{2 \pi}{3} .
$$

Then $X$ is minimal $\mathfrak{F}_{3}$-universal.

Proof. It is clear that $Y \hookrightarrow X$ holds for every $Y \in \mathfrak{M B}$ with $|Y| \leqslant 3$. Let a triangle $\{e, f, g\} \notin \mathfrak{M B}$ and let $\angle e f g$ be the maximal angle of this triangle. Suppose $\angle e f g \geqslant \frac{2 \pi}{3}$. We can locate $\{e, f, g\}$ such that $f, g \in \overrightarrow{o b}$ and $e$ lies between the rays $\overrightarrow{o a}$ and $\overrightarrow{o b}$ (see Figure 8). Doing a parallel shift of $\{e, f, g\}$ along the ray $\overrightarrow{o b}$ we can find a position of its image $\left\{e_{1}, f_{1}, g_{1}\right\}$ such that $e_{1} \in \overrightarrow{o a}$ (see Figure 8). Thus $\{e, f, g\} \hookrightarrow X$ holds. 


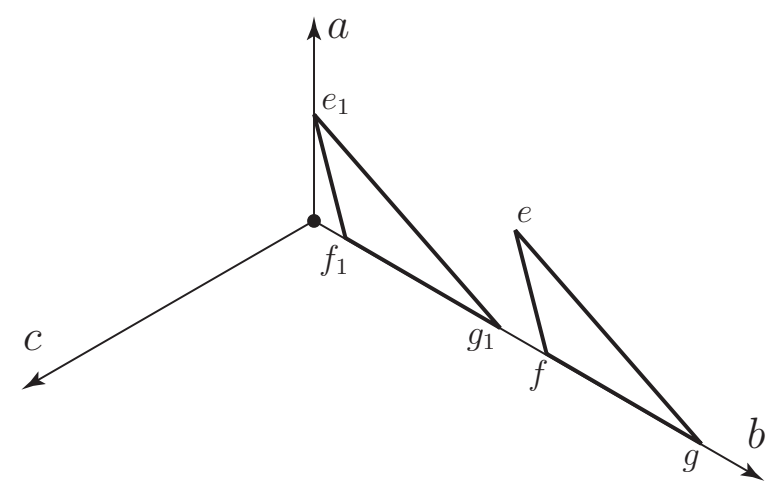

Figure 8 .

Suppose now that the maximal angle of the triangle $\{e, f, g\}$ is strictly less than $\frac{2 \pi}{3}$. Then $\{e, f, g\} \hookrightarrow X$ holds by Lemma 8.1 (see Figure 9 ). Thus $X$ is $\mathfrak{F}_{3}$-universal.

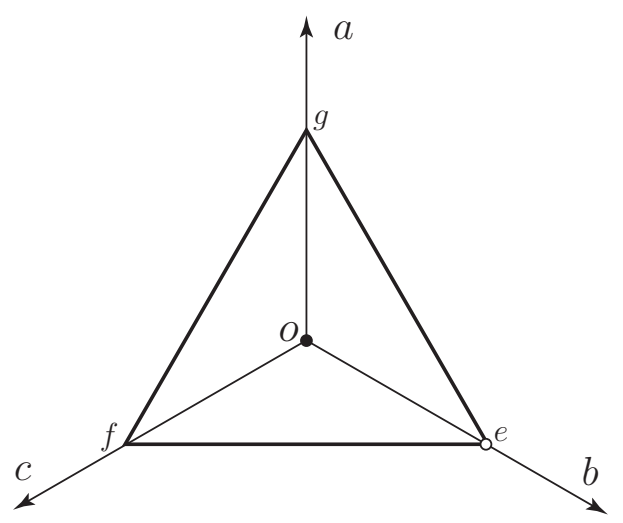

Figure 9 .

Let us prove the minimality. Let $e$ be a point of $X \backslash\{o\}$. Without loss of generality we may take $e \in \overrightarrow{o b}$ (see Figure 9). Let us consider the points $f \in \overrightarrow{o c}$ and $g \in \overrightarrow{o a}$ satisfying the equalities

$$
d_{\mathbf{R}^{2}}(o, f)=d_{\mathbf{R}^{2}}(o, g)=d_{\mathbf{R}^{2}}(o, e) .
$$

We claim that

$$
\Psi(\{e, f, g\})=\{e, f, g\}
$$

holds for every $\Psi:\{e, f, g\} \hookrightarrow X$. Indeed, it is easy to show that the sets

$$
\Psi(\{e, f, g\}) \cap(\overrightarrow{o a} \backslash\{o\}), \Psi(\{e, f, g\}) \cap(\overrightarrow{o b} \backslash\{o\}) \text { and } \Psi(\{e, f, g\}) \cap(\overrightarrow{o c} \backslash\{o\})
$$

are non-empty. Moreover, we have the equalities

$$
\angle \Psi(e) o \Psi(f)=\angle \Psi(f) o \Psi(g)=\angle \Psi(g) o \Psi(e)=\frac{2 \pi}{3} .
$$

Since $\{\Psi(e), \Psi(f), \Psi(g)\}$ is an equilateral triangle, Lemma 8.1 implies that $o$ is the incenter of $\{\Psi(e), \Psi(f), \Psi(g)\}$, i.e., the point of the intersection of the interior angle bisectors of this triangle. Consequently we have

$$
d_{\mathbf{R}^{2}}(o, \Psi(f))=d_{\mathbf{R}^{2}}(o, \Psi(g))=d_{\mathbf{R}^{2}}(o, \Psi(e)) .
$$

In view that $\{e, f, g\}$ is equilateral and that

$$
\{e, f, g\} \simeq\{\Psi(e), \Psi(f), \Psi(g)\},
$$

(8.4) follows from (8.3) and (8.5). 
Using Lemma 8.1 we see also that $\{e, f, g\} \hookrightarrow X \backslash\{o\}$ if $\{e, f, g\}$ is an triangle with an angle equal to $\frac{2 \pi}{3}$. This finishes the proof.

Theorem 8.3. Let $X_{\alpha} \subseteq \mathbf{R}^{2}$ be a set consisting of two rays $\overrightarrow{o a}$ and $\overrightarrow{o b}$ without the point $o$ and let $\alpha$ be the smaller angle between these rays. Then the metric space $X_{\alpha}$ is $\mathfrak{F}_{3}$-universal if and only if $0<\alpha<\frac{\pi}{3}$ and, in addition, $X_{\alpha}$ is minimal $\mathfrak{F}_{3}$-universal if and only if $\frac{\pi}{5} \leqslant \alpha<\frac{\pi}{3}$.

Proof. It is easy to see that in the case when $\frac{\pi}{3} \leqslant \alpha \leqslant \pi$ the inscribing of any equilateral triangle in $X_{\alpha}$ is impossible.

Let us prove the $\mathfrak{F}_{3}$-universality of $X_{\alpha}$ in the case when $0<\alpha<\frac{\pi}{3}$. For the triangles $\{e, f, g\} \in \mathfrak{M} \mathfrak{B}$, it is trivial. Now let $\{e, f, g\} \notin \mathfrak{M B}$. Without loss of generality suppose $\angle f e g \geqslant \frac{\pi}{3}$. Write $\beta=\angle f e g$ for short. Let us locate $\{e, f, g\}$ in the way depicted on Figure 10.

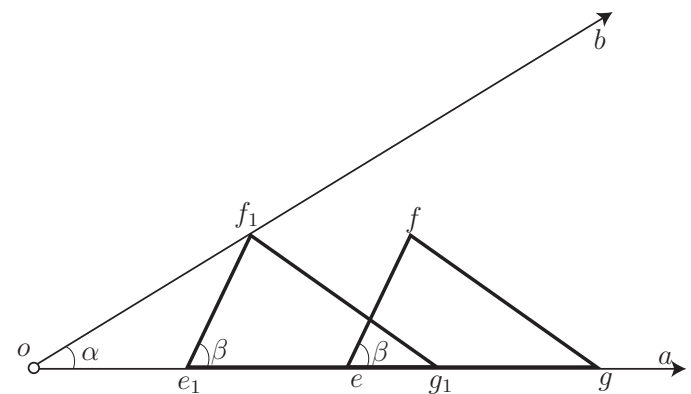

Figure 10. If $0<\alpha<\frac{\pi}{3}$, then $X_{\alpha}$ is $\mathfrak{F}_{3^{-}}$ universal.

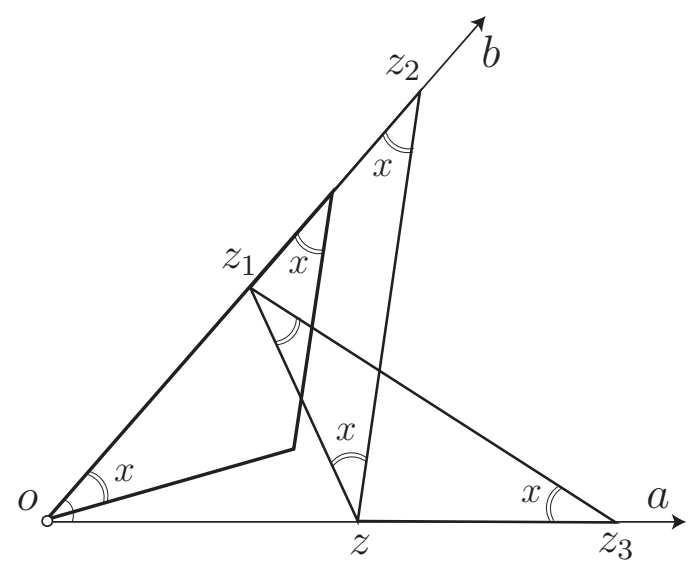

Figure 11. If $\frac{\pi}{5} \leqslant \alpha<\frac{\pi}{3}$, then $X_{\alpha}$ is minimal.

Doing a parallel shift of the $\{e, f, g\}$ along the ray $\overrightarrow{o a}$ we can find a position of its image $\left\{e_{1}, f_{1}, g_{1}\right\}$ with $e_{1} \in \overrightarrow{o b}$. Moreover, we have $e_{1} \neq o$ because $\beta>\alpha$. The desired embedding is obtained.

Let us prove that the space $X_{\alpha}$ is minimal $\mathfrak{F}_{3}$-universal for $\frac{\pi}{5} \leqslant \alpha<\frac{\pi}{3}$. It suffices to show that after deleting any point $z$ from the set $X_{\alpha}$ there exists a triangle which is not embeddable into $X_{\alpha} \backslash\{z\}$. Suppose, without loss of generality, that $z \in \overrightarrow{o a}$ (see Figure 11). Let us choose $z_{1} \in \overrightarrow{o b}$ and $z_{2} \in \overrightarrow{o b}$ such that

$$
d_{\mathbf{R}^{2}}(o, z)=d_{\mathbf{R}^{2}}\left(o, z_{1}\right) \text { and } d_{\mathbf{R}^{2}}\left(z, z_{1}\right)=d_{\mathbf{R}^{2}}\left(z_{1}, z_{2}\right) .
$$

We claim that $\left\{z, z_{1}, z_{2}\right\} \hookrightarrow X_{\alpha} \backslash\{z\}$. Let $z_{3} \in \overrightarrow{o a}$ with $d_{\mathbf{R}^{2}}\left(z, z_{1}\right)=d_{\mathbf{R}^{2}}\left(z, z_{3}\right)$. It is easy to see that $\left\{z, z_{1}, z_{2}\right\} \simeq\left\{z, z_{1}, z_{3}\right\}$. Let us show that there are no other triangles in $X_{\alpha}$ which are isometric to $\left\{z, z_{1}, z_{2}\right\}$. For the angle $x$, we have the equality $x=\frac{\pi}{4}-\frac{\alpha}{4}$ (see Figure 11). The double inequality $\frac{\pi}{5} \leqslant \alpha<\frac{\pi}{3}$ implies $\frac{\pi}{6}<x \leqslant \frac{\pi}{5}$, i.e., $x \leqslant \alpha$. The last inequality and a simple geometric reasoning show that the inscribing of $\left\{z, z_{1}, z_{2}\right\}$ into the set $X_{\alpha}$ such that the angles $x$ and $\alpha$ have the common rays $\overrightarrow{o a}$ or $\overrightarrow{o b}$ is impossible (see Figure 11).

Let us prove that $X_{\alpha}$ is not minimal if $0<\alpha<\frac{\pi}{5}$. It suffices to show that for every point $z \in X_{\alpha}$ and every triangle $\{e, f, g\}$ there are some embeddings $\{e, f, g\} \hookrightarrow$ $X_{\alpha} \backslash\{z\}$. 


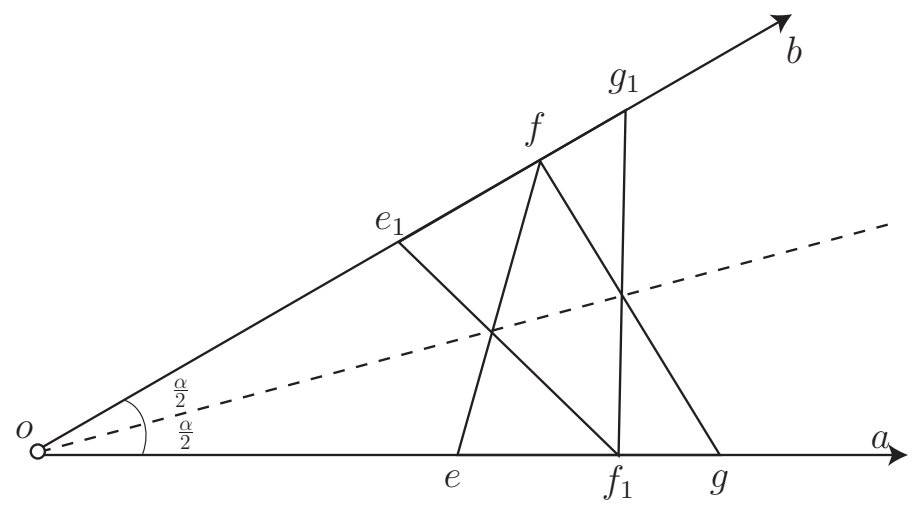

Figure 12 .

Consider first the case when $\{e, f, g\}$ is not isosceles. Without loss of generality assume $z \in \overrightarrow{o a}$. Let $\Psi:\{e, f, g\} \hookrightarrow X_{\alpha}$ be an isometric embedding with $\Psi(e)=z$ (see Figure 12).

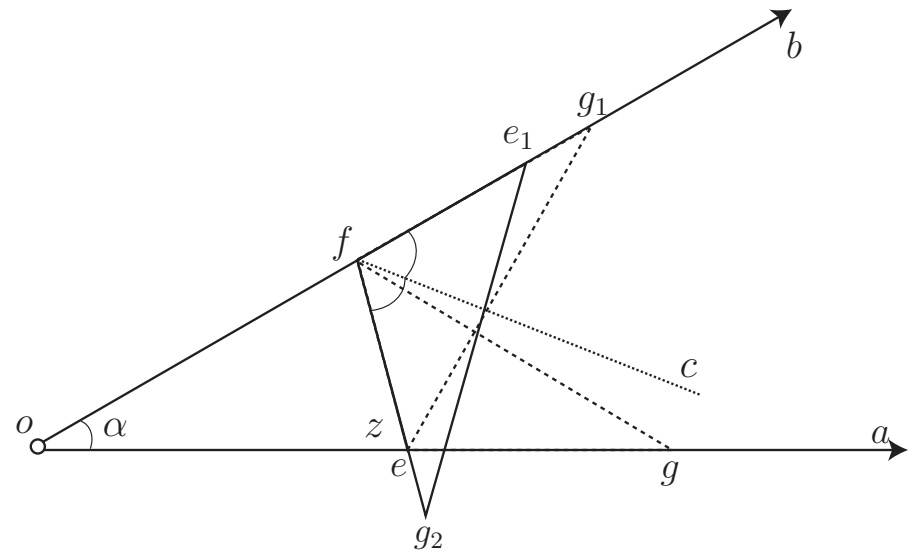

Figure 13.

Let us construct a new triangle $\left\{e_{1}, f_{1}, g_{1}\right\}$ which is symmetric to $\{\Psi(e), \Psi(f)$, $\Psi(g)\}$ with respect to the bisector of the angle $\alpha$ (see Figure 12). If $d_{\mathbf{R}^{2}}(o, \Psi(e)) \neq$ $d_{\mathbf{R}^{2}}(o, \Psi(f))$, then $f_{1}$ does not coincide with $z$ so that we have found the desirable embedding. Otherwise, let us consider the triangle $\left\{\Psi(f), g_{1}, g_{2}\right\}$ which is the reflection of $\left\{\Psi(e), \Psi(f), g_{1}\right\}$ with respect to the bisector of $\angle \Psi(e) \Psi(f) g_{1}$ (see Figure 13). Here we have $d_{\mathbf{R}^{2}}(\Psi(e), \Psi(f)) \neq d_{\mathbf{R}^{2}}\left(\Psi(f), g_{2}\right)$ because $\{e, f, g\}$ is not isosceles. Doing a parallel shift of the $\left\{\Psi(f), g_{1}, g_{2}\right\}$ along the ray $\overrightarrow{o b}$ one can find a position of its image such that the point $g_{2}$ belongs to the ray $\overrightarrow{o a}$. The desirable embedding is obtained.

Consider the case when $\{e, f, g\}$ is isosceles. Let $z \in X_{\alpha}$ and let

$$
\Psi:\{e, f, g\} \hookrightarrow X_{\alpha} \text { with } z \in\{\Psi(e), \Psi(f), \Psi(g)\} .
$$

Assume that $z \in \overrightarrow{o a}$. We are interested only in the cases when two of the vertices of $\{\Psi(e), \Psi(f), \Psi(g)\}$ are symmetric with respect to the bisector of the angle $\alpha$ and one of them coincides with $z$. Otherwise one can find a desirable embedding as above.

Let us show that in the case under consideration there is $\Psi:\{e, f, g\} \hookrightarrow X_{\alpha}$ with $z \notin\{\Psi(e), \Psi(f), \Psi(g)\}$. Further, without loss of generality, we assume that $\Psi(e)$ and $\Psi(f)$ are symmetric with respect to the bisector of $\alpha$. There exist three cases:

(i) $\Psi(g) \in \overrightarrow{o b}$ and $d_{\mathbf{R}^{2}}(o, \Psi(g))>d_{\mathbf{R}^{2}}(o, \Psi(f))$ (see Figure 14);

(ii) $\Psi(g) \in \overrightarrow{o b}$ and $d_{\mathbf{R}^{2}}(o, \Psi(g))<d_{\mathbf{R}^{2}}(o, \Psi(f))$ (see Figure 15); 
(iii) $\Psi(g) \in \overrightarrow{o a}$ and $d_{\mathbf{R}^{2}}(o, \Psi(g))<d_{\mathbf{R}^{2}}(o, \Psi(e))$ (see Figure 16).

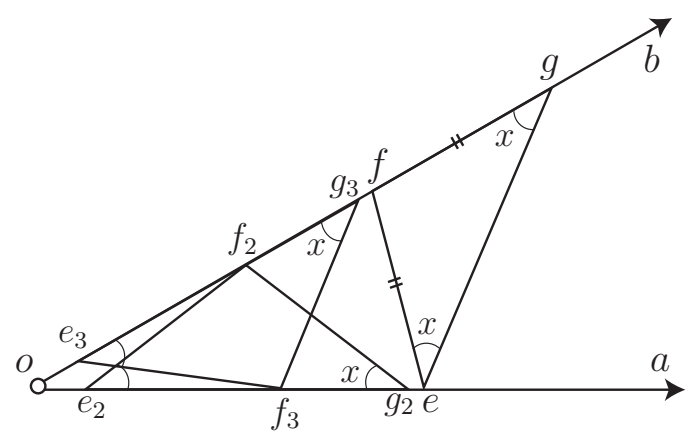

Figure 14. Case (i): $x=\frac{\pi}{4}-\frac{\alpha}{4}$.

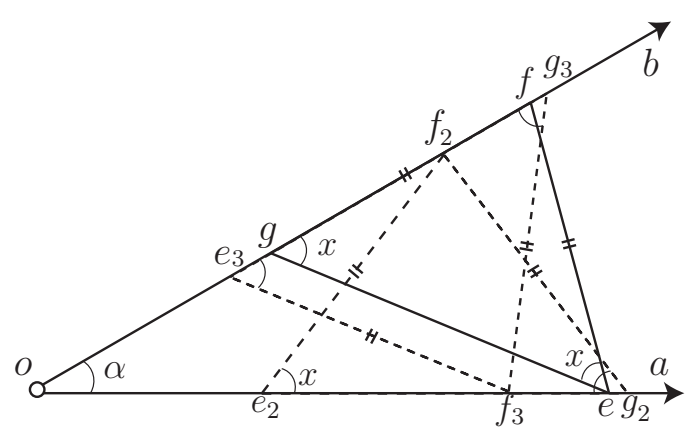

Figure 15. Case (ii): $x=\frac{\pi}{4}+\frac{\alpha}{4}$.

Consider case (i). Write $x=\angle \Psi(f) \Psi(e) \Psi(g)=\angle \Psi(f) \Psi(g) \Psi(e)$. A simple geometric reasoning gives us $x=\frac{\pi}{4}-\frac{\alpha}{4}$. If $0<\alpha<\frac{\pi}{5}$, then $\frac{\pi}{5}<x<\frac{\pi}{4}$. Hence $x>\alpha$. In virtue of the last inequality there exist $\left\{e_{2}, f_{2}, g_{2}\right\} \subseteq X_{\alpha}$ and $\left\{e_{3}, f_{3}, g_{3}\right\} \subseteq X_{\alpha}$ such that

$$
\left\{e_{2}, f_{2}, g_{2}\right\} \cap\left\{e_{3}, f_{3}, g_{3}\right\}=\varnothing \quad \text { and } \quad\left\{e_{2}, f_{2}, g_{2}\right\} \simeq\{e, f, g\} \simeq\left\{e_{3}, f_{3}, g_{3}\right\}
$$

(see Figure 14). Consequently we have $z \notin\left\{e_{2}, f_{2}, g_{2}\right\}$ or $z \notin\left\{e_{2}, f_{3}, g_{3}\right\}$. The existence of $\Psi:\{e, f, g\} \hookrightarrow X_{\alpha} \backslash\{z\}$ follows.

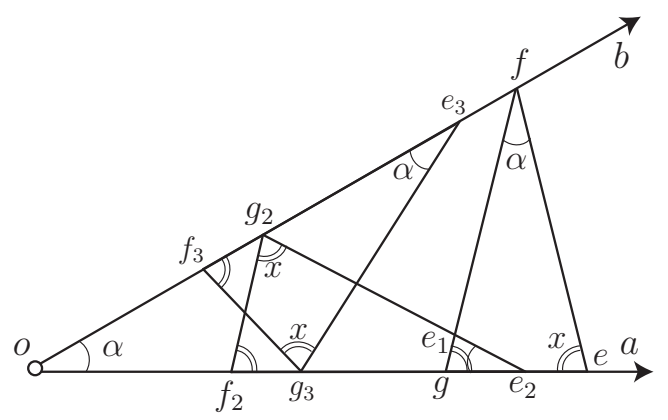

Figure 16. Case (iii): $x=\frac{\pi}{2}-\frac{\alpha}{2}$.

Cases (ii) and (iii) are similar.

There exists a simple example of a metric space $X$ which is minimal $\mathfrak{F}_{3}$-universal but not embeddable in any Hilbert space $H$,

$$
\mathfrak{F}_{3} \hookrightarrow X \hookrightarrow H .
$$

Example 8.4. Let $\overrightarrow{o a}_{i}, i=1,2,3$, be a distinct rays in $\mathbf{R}^{2}$ with the common vertex $o$ and let

$$
X=\left(\bigcup_{i=1}^{3} \overrightarrow{o a_{i}}\right) \backslash\{o\}
$$

Define a metric on $X$ as

$$
d_{X}(p, q)= \begin{cases}d_{\mathbf{R}^{2}}(p, q) & \text { if } p, q \in{\overrightarrow{o a_{i}}}_{i} \text { for } i \in\{1,2,3\} \\ d_{\mathbf{R}^{2}}(o, p)+d_{\mathbf{R}^{2}}(o, q) & \text { if } p \in{\overrightarrow{o a_{i}}}_{i}, \overrightarrow{o a_{j}}, i \neq j, \\ & \text { and } i, j \in\{1,2,3\}\end{cases}
$$


Using Lemma 1.2.1 from [7], and the definition of $d_{X}$ we can prove that $X$ is minimal $\mathfrak{F}_{3}$-universal. To prove $X \nrightarrow H$ it suffices to note that the set

$$
\bigcup_{i=1}^{3} \overrightarrow{o a_{i}}
$$

with the metric defined by (8.6) is isometric to the completion of $X$ for which $\overrightarrow{o a_{1}} \cup \overrightarrow{o a_{2}}$ and $\overrightarrow{o a_{2}} \cup \overrightarrow{o a_{3}}$ are distinct geodesic lines with the common geodesic ray $\overrightarrow{o a_{2}}$.

Acknowledgments. The authors express their gratitude to all persons and organizations which supported the present research. A grant received from TUBITAK within 2221-Fellowship Programme for Visiting Scientists on Sabbatical Leave and the hospitality of Mersin University allowed to begin these studies in Turkey in the fall of 2014. Professor Jüergen Prestin partially supported these studies as a part of EUMLS project with grant PIRSES-GA-2011-295164 in Institute of Mathematics, University of Lübeck, Germany. The research was also partially supported by grant 0115U000136 of Ministry Education and Science of Ukraine and by the State Found for Fundamental Research, Project F 71/20570. We also thank Professor Olli Martio, University of Helsinki, and Professor Matti Vuorinen, University of Turku, for the possibility to present these research on Analysis Seminar of University of Helsinki, Finland. Finally, we would like to thank the anonymous referee whose non-trivial remarks strongly helped us in preparing of the final version of this paper.

\section{References}

[1] Anderson, J. W.: Hyperbolic geometry. Second edition. - Springer-Verlag, London, 2005.

[2] Banach, S.: Théorie des opérations linéaries. - Warszawa, 1932.

[3] Berger, M.: Geometry I. - Springer-Verlag, Berlin, 1987.

[4] Bilet, V., and O. Dovgoshey: Metric betweenness, Ptolemaic spaces, and isometric embeddings of pretangent spaces in R. - J. Math. Sci. (N. Y.) 182:4, 2012, 22-36.

[5] Blumenthal, L.: Theory and applications of distance geometry. - Clarendon Press, Oxford, 1953.

[6] Bondy, J. A., and U.S. R. MurTy: Graph theory. - Springer, Berlin, 2008.

[7] Buylo, S., and V. Schroeder: Elements of asymptotic geometry. - Eur. Math. Soc., Zürich, 2007.

[8] Coxeter, M. S. M.: Introduction to geometry. Second edition. - John Wiley \& Sons, NewYork-London-Sydney, 1969.

[9] Davey, B., and H. Pristley: Introduction to lattices and order. Second edition. - Cambridge Univ. Press, Cambridge, 2002.

[10] Dovgoshei, A.A., and E. A. Petrov: Subdominant pseudoultrametric on graphs. - Sb. Math. 204:7-8, 2013, 1131-1151.

[11] Dovgoshey, O., and D. Dordovskyi: Betweenness relation and isometric imbeddings of metric spaces. - Ukrainian Math. J. 61:10, 2009, 1556-1567.

[12] Dovgoshey, O., and D. Dordovskyi: Ultrametricity and metric betweenness in tangent spaces to metric spaces. - P-Adic Numbers Ultrametric Anal. Appl. 2:2, 2010, 100-113.

[13] Dovgoshey, O., O. Martio, and M. Vuorinen: Metrization of weighted graphs. - Ann. Comb. 17, 2013, 455-476.

[14] Dress, A., and R. Scharlay: Gated sets in metric spaces. - Aequat. Math. 34:1, 1987, $112-120$. 
[15] Dutrieux, Y., and G. Lancien: Isometric embeddings of compact spaces into Banach spaces. - J. Funct. Anal. 255:2, 2007, 494-501.

[16] Fréchet, M.: Les dimensions d'un ensemble abstrait. - Math. Ann. 68, 1910, 145-168.

[17] Godefroy, G., and N. Kalton: Isometric embeddings and universal spaces. - Extr. Math. 22:2A, 2007, 179-189.

[18] Hilbert, D.: Grundlagen der Geometric. - Teubner, Leipzig, 1903.

[19] Holstynski, W.: $\mathbf{R}^{n}$ as universal metric space. - Notices Amer. Math. Soc. 25A, 1978, 367.

[20] Holstynski, W.: Metric spaces universal for 2-point spaces. - http://wlod.wordpress.com/ article/metric-spaces-universal-for-2-point-1jxfhq4x4sw0j-45.

[21] Holstynski, W.: Metric spaces universal for 3- and 4-point spaces. - http://wlod.wordpress. $\mathrm{com} /$ article/metric-spaces-universal-for-3-and-4-1jxfhq4x4sw0j-46.

[22] ILIADIs, S.: Compact and completely bounded metric spaces and isometric embeddings. - Russ. Math. Surv. 50:6, 1995, 179-180.

[23] ILIADis, S.: Isometric embeddings and universality. - Russ. Math. Surv. 48:5, 1993, 173-174.

[24] Janos, L.: A metric characterization of zero-dimensional spaces. - Proc. Amer. Math. Soc. $31: 1,1972,268-270$.

[25] Kamo, H.: Computability and computable uniqueness of Urysohn's universal metric space. In: Computability and Complexity in Analysis, 326-7/2005 of Informatik Berichte, T. Grubba, P. Hertling, H. Tsuiki and K. Weihrauch editors, FernUniversität in Hagen, Germany, 2005, $149-159$.

[26] KatĚtov. M.: On universal metric spaces. - In: General Topology and its Relations to Modern Analysis and Algebra VI, Proc. VI Symp., Prague, Czech, 1986, Res. Expo. Math. 16, 1988, $323-330$.

[27] Kuratowski, M.: Quelques problèmes concernant les espaces métriques nonseparables. Fund. Math. 25, 1935, 534-545.

[28] LANG, S.: Undergraduate algebra. Third edition. - Springer, New York, 2005.

[29] Lemin, A., and V. Lemin: On an universal ultrametric space. - Topology Appl. 103, 2000, $339-345$.

[30] LEŠNIK, D.: Constructive Urysohn universal metric space. - J. USC 15:6, 2009, 1236-1263.

[31] Martin, H. W.: Strongly rigid metrics and zero dimensionality. - Proc. Amer. Math. Soc. 67:1, 1977, 157-161.

[32] Mendris, R., and P. Zlatoš: Axiomatization and undecidability rezults for metrizable betweenness relations. - Proc. Amer. Math. Soc. 123, 1995, 873-882.

[33] Menger, K.: Untersuchungen über allgemeine Metrik I-III. - Math. Ann. 100:1, 1928, 75-163.

[34] Moszynska, M.: Theory of equidistance and betweenness relations in regular metric spaces. - Fund. Math. 96, 1977, 17-29.

[35] Papadopoulos, A.: Metric spaces, convexity and nonpositive curvature. - Eur. Math. Soc., Zürich, 2005.

[36] RADO, R.: Universal graphs and universal functions. - Acta Arith. 9, 1964, 331-340.

[37] Rodriguez-PiazzA, L.: Every separable Banach space is isometric to a space of continuous nowhere differentiable functions. - Proc. Amer. Math. Soc. 123:12, 1995, 3649-3654.

[38] SearcóId, M.: Metric spaces. - Springer, Berlin, 2006.

[39] Šımko, J.: Metrizable and R-metrizable betweenness spaces. - Proc. Amer. Math. Soc. 127, 1999, 323-325.

[40] Timan, A., and I. VestFrid: Any separable ultrametric space can be isometrically embedded in $l_{2}$. - Funct. Anal. Appl. 17:1, 1983, 70-71. 
[41] Urysohn, P.: Sur un espace métrique universel. - C. R. Acad. Sci. Paris 180, 1925, 803-806.

[42] Urysohn, P.: Sur un espace métrique universel. - Bull. Sci. Math. 51, 1927, 1-38.

[43] UspenskiJ, V.: On the group of isometries of the Urysohn universal metric space. - Comment. Math. Univ. Carolin. 31:1, 1990, 181-182.

[44] UspenskiJ, V.: Compactifications of topological groups. - In: Proceedings of the Ninth Prague Topological Symposium, Prague, August 19-25, 2001, P. Simon (ed.), Topology Atlas, 2002, $331-346$.

[45] UspenskiJ, V.: The Urysohn universal metric space is homeomorphic to a Hilbert space. Topology Appl. 139, 2004, 145-149.

[46] Vaughan, J.: Universal ultrametric space of smallest weight. - Topology Proc. 24, 1999, 611-619.

[47] Vershik, A. M.: The universal Urysohn space, Gromov's triples, and random metrics on the series of natural numbers. - Russian Math. Surveys 53:5, 1998, 921-928.

[48] Vershik, A. M.: Random metric spaces and the universal Urysohn space. - In: Fundamental Mathematics Today, 10th Anniversary of the Independent Moscow University, MCCME Publishers, 2002.

[49] Vershik, A. M.: A random metric space is a Urysohn space. - Dokl. Akad. Nauk 387:6, 2002, 733-736.

[50] Vestfrid, I.: On the universal ultrametric space. - Ukrainian Math. J. 46:12, 1994, 1890-1898.

[51] Vick, J. W.: Homology theory. An introduction to algebraic topology. Second edition. Springer-Verlag, New York, 1994.

[52] Wald, A.: Axiomatik des Zwischenbegriffers in metrischen Räumen. - Math. Ann. 104, 1931, 476-484. 